\title{
Medication adherence and persistence in patients with rheumatoid arthritis, psoriasis, and psoriatic arthritis: a systematic literature review
}

This article was published in the following Dove Press journal:

Patient Preference and Adherence

\author{
Mwangi J Murage' \\ Vanita Tongbram ${ }^{2}$ \\ Steven R Feldman ${ }^{3}$ \\ William N Malatestinic' \\ Cynthia J Larmore' \\ Talia M Muram' \\ Russel T Burge ${ }^{1,4}$ \\ Charles Bay ${ }^{2}$ \\ Nicole Johnson ${ }^{2}$ \\ Sarah Clifford ${ }^{5}$ \\ Andre B Araujo'
}

'Eli Lilly and Company, Indianapolis, IN, USA; ${ }^{2}$ ICON PIc, New York, NY, USA; ${ }^{3}$ Wake Forest University School of Medicine, Winston-Salem, NC, USA; ${ }^{4}$ University of Cincinnati, Division of Pharmaceutical Sciences, Winkle College of Pharmacy, Cincinnati, OH, USA; ${ }^{5} \mathrm{ICON} \mathrm{Plc}$, San Francisco, CA, USA
Correspondence: Mwangi J Murage Eli Lilly and Company, LCT - South Building I45-2, Drop Code 5324, I 555 Harding Street, Indianapolis, IN 4622I, USA

$\mathrm{Tel}+\mathrm{I} 3174603619$

Fax +13176516199

Email murage_mwangi_james@lilly.com
Purpose: Proper adherence and persistence to medications are crucial for better quality of life and improved outcomes in rheumatoid arthritis (RA), psoriasis ( $\mathrm{PsO}$ ), and psoriatic arthritis (PsA). We systematically describe current adherence and persistence patterns for RA, PsO, and PsA, with a focus on biologics and identifying factors associated with adherence and persistence.

Patients and methods: Using various databases, a systematic literature review of US-based studies published from 2000 to 2015 on medication adherence and persistence to biologics and associated factors was conducted among patients with RA, PsO, and PsA.

Results: Using the medication possession ratio or the percentage of days covered $>80 \%$, $\mathrm{RA}$ and $\mathrm{PsO}$ adherence rates for etanercept, adalimumab, and infliximab ranged from $16 \%$ to $73 \%, 21 \%$ to $70 \%$, and $38 \%$ to $81 \%$, respectively. Using the criteria of a $\geq 45$-day gap, RA persistence rates for etanercept, adalimumab, and infliximab ranged from $46 \%$ to $89 \%, 42 \%$ to $94 \%$, and $41 \%$ to $76 \%$, respectively. In $\mathrm{PsO}$, persistence rates for etanercept and adalimumab ranged from $34 \%$ to $50 \%$ and $50 \%$ to $62 \%$, respectively. Similar persistence rates were observed in PsA. Experienced biologics users showed better adherence and persistence. Younger age, female gender, higher out-of-pocket costs, greater disease severity, and more comorbidities were associated with lower adherence and persistence rates. Qualitative surveys revealed that nonpersistence was partly due to perceived ineffectiveness and safety/ tolerability concerns.

Conclusion: Biologic adherence and persistence rates in RA, PsO, and PsA in the United States were low, with significant opportunity for improvement. Various factors - including decrease in disease severity; reduction of comorbidities; lower out-of-pocket costs; refilling at specialty pharmacies; and awareness of drug effectiveness, safety, and tolerability - can inform targeted approaches to improve these rates.

Keywords: biologics, compliance, nonadherence, nonpersistence, factors, discontinuation

\section{Introduction}

Rheumatoid arthritis (RA), psoriasis ( $\mathrm{PsO}$ ), and psoriatic arthritis (PsA) are chronic inflammatory diseases that affect patients' social, emotional, and physical well-being. ${ }^{1-4}$ With a diagnosis rate of 41 per 100,000 people worldwide, RA affects approximately $0.5 \%$ (1.3 million) of adults in the United States, ${ }^{5}$ with women approximately 2.5 times more likely to develop RA than men. ${ }^{6}$ According to the World Psoriasis Day Consortium, $2 \%-3 \%$ of the population (125 million people worldwide) experience $\mathrm{PsO}^{7}$ and $18 \%-42 \%$ of those with PsO also have PsA. ${ }^{7}$ In the USA, in 2013, an estimated 7.4 million adults had $\mathrm{PsO}{ }^{8}$ 
Multiple therapies can treat RA, PsO, and PsA improving in health-related quality of life, relieving symptoms, slowing disease progression for RA and PsA, and providing greater clearance of PsO. However, proper adherence and persistence are crucial to realize these outcomes.

Adherence is the extent to which a patient takes a medication as prescribed by his or her health care professional and is usually reported as medication possession ratio (MPR) or percentage of days covered (PDC). ${ }^{1,9}$ Medication persistence is generally defined as the duration of time from initiation of therapy to discontinuation and is usually reported as number of days of continuous therapy use. ${ }^{9}$ Persistence can also be reported as persistence rate, or the percentage of patients continuing with treatment for a defined duration without a predefined gap in treatment. ${ }^{9}$ Adherence and persistence are problematic in RA, PsO, and PsA. ${ }^{1,2,10-12}$ Nonadherence and nonpersistence lead to suboptimal patient outcomes and place a substantial burden on the health care system. ${ }^{13}$ Health care costs attributable to nonadherence annually in the United States are estimated to be $\$ 100-\$ 300$ billion per year. ${ }^{14}$

A systematic literature review was performed to evaluate medication adherence and persistence reported among patients with RA, PsO, or PsA who are treated with biologics, and identify factors associated with adherence and persistence. There are existing systematic literature reviews of adherence and persistence rates among the individual diseases for multiple treatment types. ${ }^{1,2,4,15-19}$ The current systematic literature review adds to the available literature by evaluating the consistency in the rates of adherence and persistence across patients with RA, PsO, or PsA that has not been previously reported.

\section{Patients and methods}

A systematic literature review of medication adherence and persistence to biologics among patients with RA, PsO, or PsA was performed through electronic databases (through December 28, 2015) and e-publications ahead of print (through January 12, 2016) in PubMed and then supplemented with searches of the MEDLINE, Embase, PsycINFO, and Cochrane databases, as well as conference proceedings from the American College of Rheumatology, the American Academy of Dermatology, and the International Society for Pharmacoeconomics and Outcomes Research from 2013 to 2015 (search terms provided in Supplementary materials). Search results were evaluated in a stepwise fashion (Figure 1), with two independent reviewers screening all abstracts and then full-text articles. A third reviewer provided facilitation of reviewer discussion/disagreement. For inclusion, studies must have met the following criteria: 1) been published between 2000 and 2016; 2) utilized an observational study design (ie, prospective cohorts, retrospective cohorts, crosssectional studies, and case-control studies except where the cases and controls were prespecified to be adherent/persistent and nonadherent/nonpersistent groups); 3) been based in the United States; 4) evaluated adults aged $\geq 18$ years receiving biologic treatment for $\mathrm{RA}, \mathrm{PsO}$, or PsA; and 5) provided rates of medication adherence and/or persistence.

Additional information on reasons or factors related to nonadherence or nonpersistence included in these studies was also collated. Data from studies meeting inclusion criteria were extracted into a standardized extraction template in Microsoft Excel by a single reviewer and were validated by a second reviewer. Any studies excluded after review of the full-text article were tabulated with the reason for exclusion. A critical assessment of the included observational studies was conducted based on the questionnaire developed for the effective health program for the Agency for Healthcare Research and Quality. ${ }^{20}$

\section{Results \\ Included studies}

Forty-three primary studies were included in this systematic literature review (Figure 1). The majority of the studies utilized a retrospective design; a few are cross-sectional studies, and two are prospective studies (Tables 1 and 2). Study participants were assessed from a variety of sources but largely represented populations from claims databases or inpatient/outpatient hospital settings. Most studies reported adherence and/or persistence patterns over 1 year, with some studies using survival methods including Kaplan-Meier plots to report longer term patterns up to 5 years. The sample sizes of the included studies vary widely, ranging from 45 patients $^{35}$ to 15,834 patients, ${ }^{41}$ with most including more than 100 patients. Adherence and/or persistence in RA was examined in 26 studies, ${ }^{21-32,41} \mathrm{PsO}$ in 12 studies, ${ }^{34-40,42,44,55-57}$ and PsA in five studies. ${ }^{32,41,42,58,59} \mathrm{~A}$ few studies evaluated a mixed group of patients or data in more than one of the disease states of interest. In addition, some studies examined adherence only, some evaluated persistence only, and others reported both adherence and persistence. In the critical assessment, domains such as study limitations, directness of linkage to the evaluated intervention, consistency, precision, and reporting bias were evaluated. Overall, the studies addressed many of these elements; however, many of the questions related to bias that may stem from differences between groups 


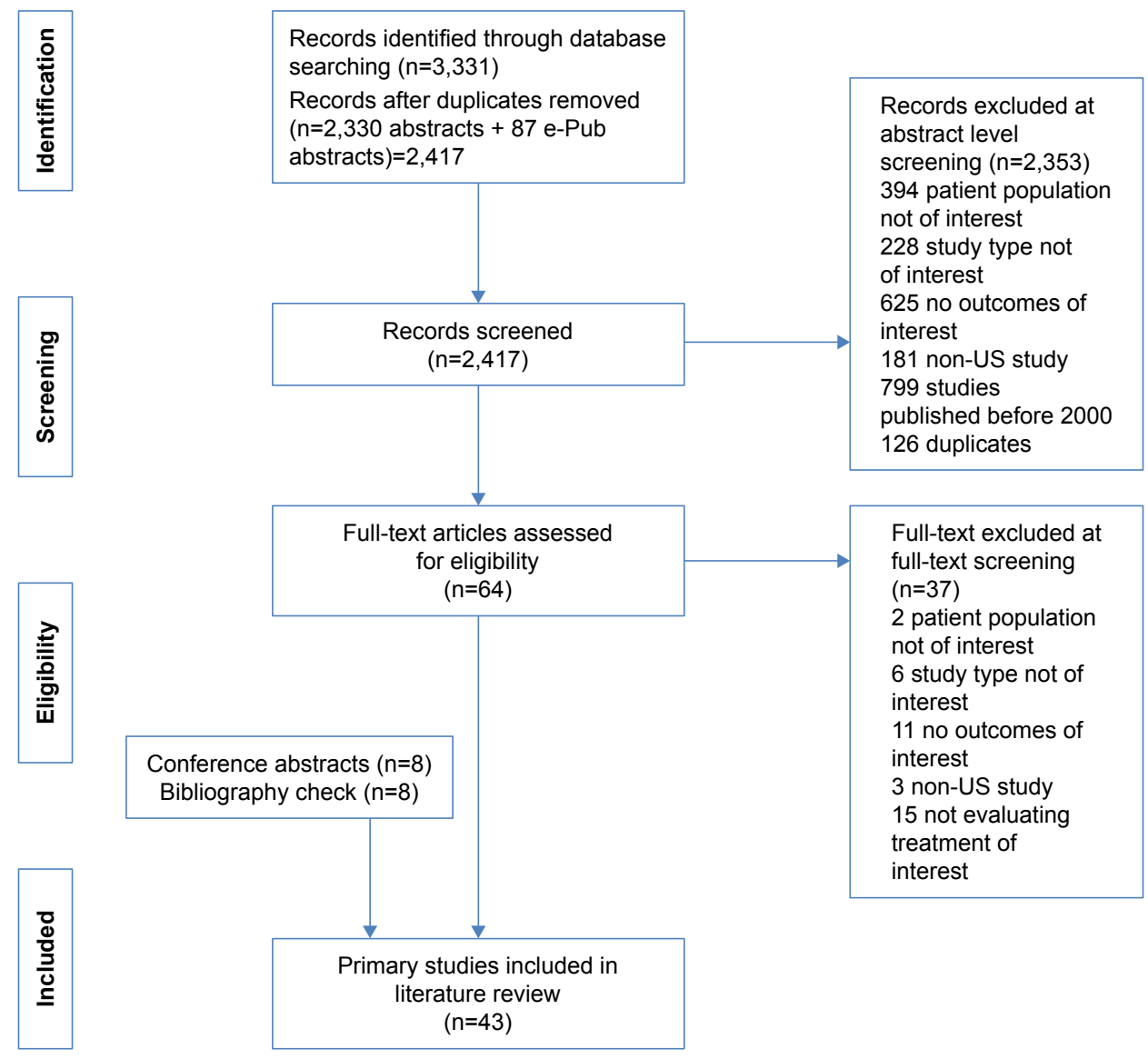

Figure I PRISMA flowchart of included studies.

Table I Medication adherence study characteristics and results

\begin{tabular}{|c|c|c|c|c|c|c|}
\hline Study & $\begin{array}{l}\text { Type of } \\
\text { study }\end{array}$ & $\begin{array}{l}\text { Source of } \\
\text { patient sample }\end{array}$ & Patient population & $\begin{array}{l}\text { Length of } \\
\text { follow-up } \\
\text { (time period) }\end{array}$ & $\begin{array}{l}\text { Adherence } \\
\text { definition }^{\mathrm{a}}\end{array}$ & $\begin{array}{l}\text { Adherence } \\
\text { rates (\%) }\end{array}$ \\
\hline \multicolumn{7}{|l|}{ RA studies } \\
\hline Chu et $\mathrm{al}^{21}$ & $\begin{array}{l}\text { Retrospective } \\
\text { cohort }\end{array}$ & KPSC-EMRs & $\begin{array}{l}\text { RA patients }(N=2,|5|) \text { : } \\
\text { ADA }(n=564), E T N \\
(n=I, 587)\end{array}$ & $\begin{array}{l}2 \text { years } \\
(2002-2009)\end{array}$ & $\mathrm{PDC} \geq 80 \%$ & $\begin{array}{l}\text { ADA (63), } \\
\text { ETN (67) }\end{array}$ \\
\hline Erhardt et $\mathrm{al}^{22}$ & $\begin{array}{l}\text { Retrospective } \\
\text { cohort }\end{array}$ & $\begin{array}{l}\text { VA clinical and } \\
\text { administrative } \\
\text { data }\end{array}$ & $\begin{array}{l}\text { RA patients }(N=2,296), \\
\text { veterans transitioning from } \\
\text { MTX monotherapy: MTX + } \\
\text { TNFi }(n=2, I 25), M T X+ \\
H C Q+S S Z(n=17 I)\end{array}$ & $\begin{array}{l}\text { I year } \\
(2006-2012)\end{array}$ & $P D C \geq 80 \%$ & $\begin{array}{l}\text { MTX + TNFi } \\
(26), M T X+ \\
\text { HCQ + SSZ (II) }\end{array}$ \\
\hline Oladapo et $\mathrm{a}^{23}$ & $\begin{array}{l}\text { Retrospective } \\
\text { cohort }\end{array}$ & Texas Medicaid & $\begin{array}{l}\text { RA patients }(\mathrm{N}=822 \text {; new } \\
\text { biologic users) within } \\
6 \text { months of injection } \\
\text { period: } E T N(n=274) \text {, ADA } \\
(n=274), \text { IFX }(n=274)\end{array}$ & $\begin{array}{l}\text { I year } \\
(2003-201 \mathrm{I})\end{array}$ & $M P R \geq 80 \%$ & 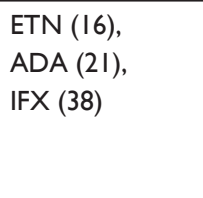 \\
\hline Tkacz et $\mathrm{al}^{24}$ & $\begin{array}{l}\text { Retrospective } \\
\text { cohort }\end{array}$ & $\begin{array}{l}\text { Optum Insight } \\
\text { Clinformatics }\end{array}$ & $\begin{array}{l}\text { RA patients }(\mathrm{N}=3,892) \text { with } \\
\text { previous prescriptions of } \\
\text { biologics: ADA }(n=I, 532) \text {, } \\
\text { ETN }(n=2,099), G O L \\
(n=26 I)\end{array}$ & $\begin{array}{l}\text { I year } \\
(2009-201 \mathrm{I})\end{array}$ & $\begin{array}{l}\text { MPR } \geq 80 \% \text { and } \\
P D C \geq 80 \%\end{array}$ & $\begin{array}{l}\text { ADA: MPR } \\
\text { (7I), PDC (70); } \\
\text { ETN: MPR (62), } \\
\text { PDC (6I); } \\
\text { GOL: MPR (82), } \\
\text { PDC (8I) }\end{array}$ \\
\hline
\end{tabular}

(Continued) 
Table I (Continued)

\begin{tabular}{|c|c|c|c|c|c|c|}
\hline Study & $\begin{array}{l}\text { Type of } \\
\text { study }\end{array}$ & $\begin{array}{l}\text { Source of } \\
\text { patient sample }\end{array}$ & Patient population & $\begin{array}{l}\text { Length of } \\
\text { follow-up } \\
\text { (time period) }\end{array}$ & $\begin{array}{l}\text { Adherence } \\
\text { definition }^{\mathrm{a}}\end{array}$ & $\begin{array}{l}\text { Adherence } \\
\text { rates }(\%)\end{array}$ \\
\hline Li et $\mathrm{al}^{25}$ & $\begin{array}{l}\text { Retrospective } \\
\text { cohort }\end{array}$ & MAX files & $\begin{array}{l}\text { RA patients }(N=2,638 ; \\
\text { new users of biologics): } \\
\text { ETN }(n=I, 359) \text {, } \\
\text { ANA }(n=267) \text {, } \\
\text { IFX }(n=I, 0 \mid 2)\end{array}$ & $\begin{array}{l}\text { I year } \\
(2000-2002)\end{array}$ & $\mathrm{PDC} \geq 80 \%$ & 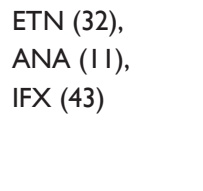 \\
\hline Borah et $\mathrm{al}^{26}$ & $\begin{array}{l}\text { Retrospective } \\
\text { cohort }\end{array}$ & $\begin{array}{l}\text { Managed care } \\
\text { administrative } \\
\text { claims data }\end{array}$ & $\begin{array}{l}\text { RA patients }(\mathrm{N}=3,829) \text {, } \\
\text { including new/experienced } \\
\text { biologic users: } \\
\text { ETN }(n=2,537) \text {, } \\
\text { ADA }(n=I, 292)\end{array}$ & $\begin{array}{l}\text { I year } \\
(2005-2006)\end{array}$ & $M P R \geq 80 \%$ & $\begin{array}{l}\text { ETN: new } \\
\text { users (65), } \\
\text { experienced } \\
\text { users (73); } \\
\text { ADA: new } \\
\text { users (63), } \\
\text { experienced } \\
\text { users (70) }\end{array}$ \\
\hline Grijalva et $\mathrm{al}^{27}$ & $\begin{array}{l}\text { Retrospective } \\
\text { cohort }\end{array}$ & $\begin{array}{l}\text { Tennessee } \\
\text { Medicaid } \\
\text { managed-care } \\
\text { program }\end{array}$ & $\begin{array}{l}\text { RA patients }(\mathrm{N}=14,586) \\
\text { with previous therapeutic } \\
\text { treatment (MTX, ETN, IFX, } \\
\text { ADA) }\end{array}$ & $\begin{array}{l}\text { NR } \\
(1995-2005)\end{array}$ & $\begin{array}{l}\text { Median MPR: The } \\
\text { percent of person- } \\
\text { time exposed to } \\
\text { initial regimen } \\
\text { during the episodes, } \\
\text { as calculated for } \\
\text { patients with } 180+ \\
\text { person-days of } \\
\text { follow-up }\end{array}$ & $\begin{array}{l}\text { Median } \\
\text { adherence: MTX } \\
(59), \text { ADA (72), } \\
\text { ETN (73), IFX } \\
(68)\end{array}$ \\
\hline Harley et $\mathrm{a}^{28}$ & $\begin{array}{l}\text { Retrospective } \\
\text { cohort }\end{array}$ & $\begin{array}{l}\text { Large US health } \\
\text { plan }\end{array}$ & $\begin{array}{l}\text { RA patients }(\mathrm{N}=2,662) \\
\text { receiving new biologic } \\
\text { or new MTX treatment } \\
\text { I } 82 \text { days prior to study } \\
\text { index: } \operatorname{MTX}(\mathrm{n}=1,668) \text {, } \\
\text { biologics (IFX }[\mathrm{n}=141] \text {, } \\
\text { ETN }[\mathrm{n}=853])\end{array}$ & I year (NR) & $\begin{array}{l}\text { Compliance } \\
\text { ratio } \geq 80 \% \text { : } \\
\text { number of therapy } \\
\text { administrations or } \\
\text { filled prescriptions } \\
\text { divided by the } \\
\text { expected number }\end{array}$ & $\begin{array}{l}\operatorname{IFX}(8 \mathrm{I}) \\
\operatorname{ETN}(68), \\
\operatorname{MTX}(64)\end{array}$ \\
\hline $\begin{array}{l}\text { Curkendall } \\
\text { et } \mathrm{al}^{29}\end{array}$ & $\begin{array}{l}\text { Retrospective } \\
\text { cohort }\end{array}$ & $\begin{array}{l}\text { MEDSTAT } \\
\text { MarketScan } \\
\text { Commercial } \\
\text { Insurance } \\
\text { claims }\end{array}$ & $\begin{array}{l}\text { Newly initiated RA patients } \\
(\mathrm{N}=2,285 ; \mathrm{ADA}, \mathrm{ETN})\end{array}$ & $\begin{array}{l}\text { I year } \\
(2002-2004)\end{array}$ & Average MPR & $\begin{array}{l}\text { Mean adherence } \\
(52)\end{array}$ \\
\hline Grijalva et $\mathrm{al}^{30}$ & $\begin{array}{l}\text { Retrospective } \\
\text { cohort }\end{array}$ & $\begin{array}{l}\text { Tennessee } \\
\text { Medicaid } \\
\text { managed-care } \\
\text { program }\end{array}$ & $\begin{array}{l}\text { RA patients }(\mathrm{N}=6,0 \mathrm{I} 8) \text { with } \\
\text { previously filled regimen and } \\
\text { a year of continuous health } \\
\text { plan enrollment: DMARDs } \\
\text { (MTX, HCQ, SSZ, LF) and } \\
\text { biologics and MTX (IFX, } \\
\text { ETN, ADA, ANA) }\end{array}$ & $\begin{array}{l}\text { I year } \\
(1995-2004)\end{array}$ & Average MPR & $\begin{array}{l}\text { Mean } \\
\text { adherence for } \\
\text { MTX (80), IFX } \\
(90), \text { MTX + } \\
\text { IFX (66), ETN } \\
(83), \text { MTX + } \\
\text { ETN (64), ADA } \\
\text { (85), MTX + } \\
\text { ADA (72) }\end{array}$ \\
\hline Barlow et $\mathrm{al}^{31}$ & $\begin{array}{l}\text { Retrospective } \\
\text { cohort }\end{array}$ & $\begin{array}{l}\text { Prescription } \\
\text { benefit plans of } \\
\text { Medco Health } \\
\text { Solutions, Inc. }\end{array}$ & $\begin{array}{l}\text { RA patients }(\mathrm{N}=4,388) \\
\text { with one previous } \\
\text { prescription of assigned } \\
\text { biologic and continuous } \\
\text { drug/medical benefit } \\
\text { (ETN, ADA): Group } \\
\text { I - specialty pharmacy } \\
(\mathrm{n}=3,054) \text {; Group } \\
2-\text { retail pharmacy } \\
(\mathrm{n}=1,334)\end{array}$ & $\begin{array}{l}\text { I year } \\
(2006-2008)\end{array}$ & $\begin{array}{l}\text { Average MPR: } \\
\text { Defined as the } \\
\text { proportion of } \\
\text { days covered by } \\
\text { medication supply } \\
\text { during the I-year } \\
\text { fixed interval }\end{array}$ & $\begin{array}{l}\text { Mean adherence } \\
\text { for Group I, } \\
\text { years I-3: } \\
(63,68,61) \text {; } \\
\text { Group 2, } \\
\text { years I-3 } \\
(50,5 I, 44)\end{array}$ \\
\hline
\end{tabular}


Table I (Continued)

\begin{tabular}{|c|c|c|c|c|c|c|}
\hline Study & $\begin{array}{l}\text { Type of } \\
\text { study }\end{array}$ & $\begin{array}{l}\text { Source of } \\
\text { patient sample }\end{array}$ & Patient population & $\begin{array}{l}\text { Length of } \\
\text { follow-up } \\
\text { (time period) }\end{array}$ & $\begin{array}{l}\text { Adherence } \\
\text { definition }^{\mathrm{a}}\end{array}$ & $\begin{array}{l}\text { Adherence } \\
\text { rates (\%) }\end{array}$ \\
\hline Ellis et $\mathrm{al}^{32}$ & $\begin{array}{l}\text { Retrospective } \\
\text { cohort }\end{array}$ & Truven database & $\begin{array}{l}\text { RA patients }(\mathrm{N}=\mathrm{I}, 036) \\
\text { with first or most recent } \\
\text { prescription for } \mathrm{GOL}\end{array}$ & $\begin{array}{l}\text { NR } \\
(2008-2010)\end{array}$ & $\begin{array}{l}\text { The number of refills } \\
\text { occurring between } 21 \\
\text { and } 38 \text { days from a } \\
\text { previous fill divided by } \\
\text { the total refill intervals }\end{array}$ & GOL (79) \\
\hline Stockl et $\mathrm{al}^{33}$ & $\begin{array}{l}\text { Prospective } \\
\text { cohort }\end{array}$ & $\begin{array}{l}\text { PBM DTM } \\
\text { program }\end{array}$ & $\begin{array}{l}\text { RA patients }(\mathrm{N}=5 \text { I } 8 \text { ) on } \\
\text { injectable RA medications } \\
(\mathrm{ETN}, \mathrm{ADA}, \mathrm{ANA}, \mathrm{ABA} \text {, } \\
\text { IFX, RIT): Group I - study } \\
\text { ITT ( } \mathrm{n}=340) \text {; Group } 2 \text { - } \\
\text { study completer ( } \mathrm{n}=244) \text {; } \\
\text { Group } 3 \text { - specialty } \\
\text { pharmacy-matched cohort } \\
\text { ( } \mathrm{n}=244) \text {; Group } 4 \text { - } \\
\text { community pharmacy- } \\
\text { matched cohort }(\mathrm{n}=244)^{\mathrm{b}}\end{array}$ & $\begin{array}{l}0.67 \text { year } \\
(2007-2008)\end{array}$ & $\begin{array}{l}\text { Mean PDC: The sum } \\
\text { of the days' supply } \\
\text { for all claims during } \\
\text { the post-identification } \\
\text { period divided by } 240 \\
\text { days }\end{array}$ & $\begin{array}{l}\text { Mean PDC: } \\
\text { Group I (83), } \\
\text { Group } 2 \text { (89), } \\
\text { Group } 3(8 I), \\
\text { Group } 4 \text { (60) }\end{array}$ \\
\hline $\begin{array}{l}\text { Bonafede } \\
\text { et } \mathrm{al}^{34}\end{array}$ & $\begin{array}{l}\text { Retrospective } \\
\text { cohort }\end{array}$ & $\begin{array}{l}\text { Truven Health } \\
\text { MarketScan } \\
\text { Commercial } \\
\text { Claims and } \\
\text { Encounters }\end{array}$ & $\begin{array}{l}\text { RA patients }(\mathrm{N}=4,542) \text { : } \\
\text { ETN + MTX }(n=3,724), \\
\text { MTX + HCQ + SSZ }(n=8 \mid 8)\end{array}$ & $\begin{array}{l}\text { I year } \\
(2009-2013)\end{array}$ & $\mathrm{PDC} \geq 80 \%$ & $\begin{array}{l}\text { ETN + MTX } \\
\text { (28), ETN alone } \\
\text { (50), MTX alone } \\
\text { (45), MTX + } \\
\text { HCQ + SSZ } \\
(18)\end{array}$ \\
\hline \multicolumn{7}{|l|}{ PsO studies } \\
\hline Sandoval et al ${ }^{35}$ & $\begin{array}{l}\text { Retrospective } \\
\text { cohort }\end{array}$ & $\begin{array}{l}\text { Wake Forest } \\
\text { School of } \\
\text { Medicine } \\
\text { Department of } \\
\text { Dermatology } \\
\text { Clinic }\end{array}$ & $\begin{array}{l}\text { PsO patients ( } N=45): \text { UST } \\
45 \mathrm{mg}(\mathrm{n}=26), \text { UST } 90 \mathrm{mg} \\
(\mathrm{n}=19)\end{array}$ & $\begin{array}{l}\text { NR } \\
(2009-2013)\end{array}$ & $\begin{array}{l}\text { Proportion of } \\
\text { injections within } \\
\pm 2 \text {-week window of } \\
\text { the recommended } \\
\text { dosing schedule } \\
\text { calculated every } \\
12 \text { weeks }\end{array}$ & $\begin{array}{l}\text { UST } 45 \mathrm{mg} \\
\text { median }(100) \text {, } \\
\text { mean }(87) ; \\
\text { UST } 90 \mathrm{mg} \\
\text { median }(80) \text {, } \\
\text { mean }(78)\end{array}$ \\
\hline Doshi et $\mathrm{al}^{36}$ & $\begin{array}{l}\text { Retrospective } \\
\text { cohort }\end{array}$ & Medicare files & $\begin{array}{l}\text { PsO patients }(N=2,707) \text { : IFX } \\
(n=3 \mid 8), \text { UST }(n=280), \text { ADA } \\
(n=1,084), \text { ETN }(n=I, 025)\end{array}$ & $\begin{array}{l}\text { I year } \\
(2009-2012)\end{array}$ & $\mathrm{PDC} \geq 80 \%$ & $\begin{array}{l}\text { IFX (49), UST } \\
\text { (43), ADA (4I), } \\
\text { ETN (29) }\end{array}$ \\
\hline Bhosle et $\mathrm{al}^{37}$ & $\begin{array}{l}\text { Retrospective } \\
\text { cohort }\end{array}$ & $\begin{array}{l}\text { North Carolina } \\
\text { Medicaid }\end{array}$ & $\begin{array}{l}\text { PsO patients }(\mathrm{N}=186) \\
\text { prescribed biologic during } \\
\text { study period (ALE, EFA, } \\
\text { ETN) }\end{array}$ & $\begin{array}{l}0.50 \text { year } \\
(200 \mathrm{I}-2004)\end{array}$ & $\begin{array}{l}\text { Proportion of days of } \\
\text { supply for dispensed } \\
\text { prescription } \\
\text { medications vs usage }\end{array}$ & $\begin{array}{l}\text { ALE (60), EFA } \\
(73), \text { ETN (68) }\end{array}$ \\
\hline Li et a $\left.\right|^{38}$ & $\begin{array}{l}\text { Retrospective } \\
\text { cohort }\end{array}$ & $\begin{array}{l}\text { Truven Health } \\
\text { MarketScan } \\
\text { Commercial } \\
\text { Claims and } \\
\text { Encounter and } \\
\text { the Medicare } \\
\text { Supplemental and } \\
\text { Coordination of } \\
\text { Benefits databases }\end{array}$ & $\begin{array}{l}\text { PsO patients }(\mathrm{N}=3,249) \\
\text { prescribed biologics } \\
\text { between the study period } \\
\text { dates: divided by PDC } \\
\text { patterns, Group I: } \\
50 \% \leq \text { PDC }<75 \% \\
\text { ( } n=760) \text {; Group 2: PDC } \\
<25 \% \text { ( } n=535) \text {; Group 3: } \\
25 \% \leq \text { PDC }<50 \% \text { ( } n=574) \text {; } \\
\text { Group 4: PDC }>75 \% \\
\text { ( } n=1,380)\end{array}$ & $\begin{array}{l}\text { I year } \\
(2007-201 \mathrm{I})\end{array}$ & $\begin{array}{l}\text { PDC-based } \\
\text { categorizations }\end{array}$ & $\begin{array}{l}\text { Continually } \\
\text { high adherence, } \\
\text { Groups I-4: } \\
(6,0,0,96) \text {; } \\
\text { High then low } \\
\text { adherence, } \\
\text { Groups I-4: } \\
(24, \text { I, 58, 0); } \\
\text { Moderate then } \\
\text { low adherence, } \\
\text { Groups I-4: } \\
\text { (0, 98, I7, 0); } \\
\text { Consistently } \\
\text { moderate } \\
\text { adherence, } \\
\text { Groups I-4: } \\
(7 I, I, 25,4)\end{array}$ \\
\hline
\end{tabular}

(Continued) 
Table I (Continued)

\begin{tabular}{|c|c|c|c|c|c|c|}
\hline Study & $\begin{array}{l}\text { Type of } \\
\text { study }\end{array}$ & $\begin{array}{l}\text { Source of } \\
\text { patient sample }\end{array}$ & Patient population & $\begin{array}{l}\text { Length of } \\
\text { follow-up } \\
\text { (time period) }\end{array}$ & $\begin{array}{l}\text { Adherence } \\
\text { definition }^{\mathrm{a}}\end{array}$ & $\begin{array}{l}\text { Adherence } \\
\text { rates (\%) }\end{array}$ \\
\hline Goren et $\mathrm{al}^{39}$ & $\begin{array}{l}\text { Web-based } \\
\text { survey }\end{array}$ & $\begin{array}{l}\text { US claims } \\
\text { database: } \\
\text { Diplomat Specialty } \\
\text { Pharmacy }\end{array}$ & $\begin{array}{l}\text { PsO patients }(\mathrm{N}=345) \\
\text { prescribed biologic between } \\
\text { study period dates: } \\
\text { ADA }(n=262), \text { UST }(n=83)\end{array}$ & $\begin{array}{l}\text { NR } \\
(2009-2011)\end{array}$ & $\begin{array}{l}\text { Adherence: number } \\
\text { of patients who } \\
\text { never missed taking } \\
\text { medication or injection }\end{array}$ & $\begin{array}{l}\text { ADA (67), } \\
\text { UST (88) }\end{array}$ \\
\hline $\begin{array}{l}\text { Kamangar } \\
\text { et } \mathrm{al}^{40}\end{array}$ & $\begin{array}{l}\text { Cross- } \\
\text { sectional }\end{array}$ & UCSF PsO Center & $\begin{array}{l}\text { PsO patients }(\mathrm{N}=\mathrm{I} 06) \\
\text { previously treated with one } \\
\text { or more biologic agents } \\
\text { (ETN, ADA, ALE, EFA, IFX, } \\
\text { UST, GOL) }\end{array}$ & $\begin{array}{l}\text { NR } \\
(2010-2011)\end{array}$ & $\begin{array}{l}\text { Frequency of a } \\
\text { patient missing a dose } \\
\text { of biologic }\end{array}$ & $\begin{array}{l}\text { Never/rarely } \\
\text { missed (67), } \\
\text { sometimes (27), } \\
\text { often (6) }\end{array}$ \\
\hline \multicolumn{7}{|l|}{ PsA study } \\
\hline Ellis et $\mathrm{al}^{32}$ & $\begin{array}{l}\text { Retrospective } \\
\text { cohort }\end{array}$ & Truven database & $\begin{array}{l}\text { PsA patients }(\mathrm{N}=325) \\
\text { with first or most recent } \\
\text { prescription for } \mathrm{GOL}\end{array}$ & $\begin{array}{l}\text { NR } \\
(2008-2010)\end{array}$ & $\begin{array}{l}\text { Proportion of the } \\
\text { number of refills } \\
\text { occurring between } 21 \\
\text { and } 38 \text { days from a } \\
\text { previous fill divided by } \\
\text { the total refill intervals }\end{array}$ & GOL (76) \\
\hline
\end{tabular}

Notes: anless specified otherwise, MPR is based on the sum of days' supply of the index treatment divided by duration of treatment. The $80 \%$ cutoff has been examined as an acceptable threshold in multiple studies assessing medication adherence. Unless specified otherwise, PDC is the percentage of days covered, based on days' supply of prescription claims during which a patient has medication available during the post-index period. The $80 \%$ cutoff has been examined in multiple studies assessing medication

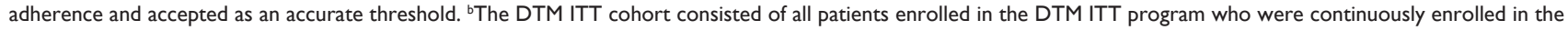
plan for the 4-month preidentification period and 8-month postidentification period. The DTM completer cohort consists of the subset of DTM ITT patients who completed the month 6 DTM consultation and who could be matched to patients in the community pharmacy and specialty pharmacy cohorts. The community pharmacy and specialty pharmacy cohorts consist of continuously enrolled patients who could be matched to the DTM completer patients.

Abbreviations: ABA, abatacept; ADA, adalimumab; ALE, alefacept; ANA, anakinra; DMARDs, disease-modifying antirheumatic drugs; DTM, drug therapy management; EFA, efalizumab; EMR, electronic medical record; ETN, etanercept; GOL, golimumab; HCQ, hydroxychloroquine; IFX, infliximab; ITT, intent-to-treat; KPSC, Kaiser Permanente Southern California; LF, leflunomide; MAX, Medicaid Analytic Extract; MPR, medication possession ratio; MTX, methotrexate; NR, not reported; PBM, pharmacy benefits management; PDC, percentage of days covered; PsA, psoriatic arthritis; PsO, psoriasis; RA, rheumatoid arthritis; RIT, rituximab; SSZ, sulfasalazine; TNFi, tumor necrosis factor alpha inhibitor; UCSF, University of California-San Francisco; US, United States; UST, ustekinumab; VA, Veterans Affairs.

Table 2 Medication persistence study characteristics and results

\begin{tabular}{|c|c|c|c|c|c|c|}
\hline Study & $\begin{array}{l}\text { Type of } \\
\text { study }\end{array}$ & $\begin{array}{l}\text { Source of } \\
\text { patient sample }\end{array}$ & Patient population & $\begin{array}{l}\text { Time } \\
\text { period }\end{array}$ & Persistence definition & $\begin{array}{l}\text { Persistence } \\
\text { rates (\%) }\end{array}$ \\
\hline \multicolumn{7}{|c|}{ RA studies } \\
\hline $\begin{array}{l}\text { Shim } \\
\text { et a }\left.\right|^{41}\end{array}$ & $\begin{array}{l}\text { Cross- } \\
\text { sectional }\end{array}$ & $\begin{array}{l}\text { Proprietary } \\
\text { administrative } \\
\text { claims database }\end{array}$ & $\begin{array}{l}\text { RA/PsA patients }(\mathrm{N}=15,834) \\
\text { with a biologic insurance } \\
\text { claim on TOC, CER, } \\
\text { ETN, ADA, and INF with } \\
\text { continuous enrollment in } \\
\text { health plan from index claim } \\
\text { to } 15 \text { months post-claim }\end{array}$ & $\begin{array}{l}\text { I year } \\
(2012-2013)\end{array}$ & $\begin{array}{l}\text { Persistent patients are those } \\
\text { with treatment length of at least } \\
365 \text { days after the index claim } \\
\text { without a therapy gap or switch } \\
\text { in therapy }\end{array}$ & Overall (67) \\
\hline $\begin{array}{l}\text { Erhardt } \\
\text { et } \mathrm{al}^{22}\end{array}$ & $\begin{array}{l}\text { Retrospective } \\
\text { cohort }\end{array}$ & $\begin{array}{l}\text { VA clinical and } \\
\text { administrative } \\
\text { data }\end{array}$ & $\begin{array}{l}\text { RA patients }(\mathrm{N}=2,296) \text {; } \\
\text { veterans transitioning from } \\
\text { MTX monotherapy: } \\
\text { MTX + TNFi }(n=2, I 25), \\
M T X+H C Q+S S Z(n=|7|)\end{array}$ & $\begin{array}{l}\text { I year } \\
(2006-2012)\end{array}$ & $\begin{array}{l}\text { Persistent patients are those } \\
\text { for whom the treatment } \\
\text { continued over the } 12 \text {-month } \\
\text { period without a } \geq 90 \text {-day gap in } \\
\text { refilling of drug }\end{array}$ & $\begin{array}{l}\text { MTX + TNFi } \\
\text { (45), MTX+ } \\
\text { HCQ + SSZ } \\
\text { (18) }\end{array}$ \\
\hline Li et $\mathrm{al}^{25}$ & $\begin{array}{l}\text { Retrospective } \\
\text { cohort }\end{array}$ & MAX files & $\begin{array}{l}\text { RA patients ( } N=2,638 \text {; new } \\
\text { users of biologics): ETN } \\
\text { ( } n=I, 359) \text {, ANA ( } n=267) \text {, } \\
\text { IFX }(n=I, 012)\end{array}$ & $\begin{array}{l}\text { I year } \\
(2000-2002)\end{array}$ & $\begin{array}{l}\text { Persistent patients are those who } \\
\text { did not have a } 90 \text {-day continuous } \\
\text { gap in therapy or switched from } \\
\text { initiation of second biologic } \\
\text { within } 90 \text { days of discontinuation } \\
\text { date of index biologic and no } \\
\text { evidence use of the second } \\
\text { (switched to) biologic in } 120 \\
\text { days before discontinuation of } \\
\text { indexed biologic }\end{array}$ & $\begin{array}{l}\text { ETN (53.8), } \\
\text { ANA (7), } \\
\text { IFX (53.7) }\end{array}$ \\
\hline
\end{tabular}


Table 2 (Continued)

\begin{tabular}{|c|c|c|c|c|c|c|}
\hline Study & $\begin{array}{l}\text { Type of } \\
\text { study }\end{array}$ & $\begin{array}{l}\text { Source of } \\
\text { patient sample }\end{array}$ & Patient population & $\begin{array}{l}\text { Time } \\
\text { period }\end{array}$ & Persistence definition & $\begin{array}{l}\text { Persistence } \\
\text { rates (\%) }\end{array}$ \\
\hline $\begin{array}{l}\text { Grijalva } \\
\text { et }\left.a\right|^{30}\end{array}$ & $\begin{array}{l}\text { Retrospective } \\
\text { cohort }\end{array}$ & $\begin{array}{l}\text { Tennessee } \\
\text { Medicaid } \\
\text { managed care } \\
\text { program }\end{array}$ & $\begin{array}{l}\text { RA patients }(\mathrm{N}=6,018) \text { with } \\
\text { previously filled regimen and } \\
\text { a year of continuous health } \\
\text { plan enrollment; biologics } \\
\text { and MTX: IFX, ETN, ADA, } \\
\text { ANA }\end{array}$ & $\begin{array}{l}\text { NR (1995- } \\
2004)\end{array}$ & $\begin{array}{l}\text { Median persistence: time to } \\
\text { cessation of therapy for at least } \\
90 \text { days without the addition } \\
\text { of an alternate DMARD and } \\
\text { time to switching to a different } \\
\text { DMARD regimen }\end{array}$ & $\begin{array}{l}\text { In months: } \\
\text { IFX (2.8), } \\
\text { ETN (5.8), } \\
\text { ADA (4.4); in } \\
\text { months: }{ }^{\mathrm{a}} \text { MTX + } \\
\text { IFX (5.I), } \\
\text { MTX + ETN } \\
(4.8), \text { MTX + } \\
\text { ADA (7.2), } \\
\text { MTX + } \\
\text { ANA (5.I) }\end{array}$ \\
\hline $\begin{array}{l}\text { Sauer } \\
\text { et } a^{42}\end{array}$ & $\begin{array}{l}\text { Retrospective } \\
\text { cohort }\end{array}$ & VHA & $\begin{array}{l}\text { RA patients }(N=|0,7| 9) \\
\text { with one biologic } \\
\text { prescription during study } \\
\text { period (new/experienced } \\
\text { biologic users); new biologic } \\
\text { users: ETN }(n=2,109), \\
\text { ADA ( } n=2,035), \text { IFX } \\
(n=263), \text { RIT ( } n=277) \text {, } \\
\text { ABA ( } n=25), G O L(n=20) \text {; } \\
\text { biologic experienced: ETN } \\
(n=3,040), \text { ADA }(n=2,120), \\
\text { IFX }(n=498), \operatorname{RIT~}(n=92), \\
\text { ABA }(n=122), G O L(n=2)\end{array}$ & $\begin{array}{l}\text { I year } \\
(2008-2011)\end{array}$ & $\begin{array}{l}\text { Persistent patients are those } \\
\text { with no } 45 \text {-day gap in index } \\
\text { biologic therapy after the end of } \\
\text { clinical benefit from the previous } \\
\text { dose and who did not switch to } \\
\text { another biologic }\end{array}$ & $\begin{array}{l}\text { RA new } \\
\text { biologic users: } \\
\text { ETN (54), ADA } \\
\text { (55), IFX (57), } \\
\text { RIT (29), ABA } \\
\text { (5I), GOL (55); } \\
\text { RA biologic } \\
\text { experienced: } \\
\text { ETN (6I), ADA } \\
\text { (6I), IFX (76), } \\
\text { RIT (38), ABA } \\
\text { (62), GOL (0) }\end{array}$ \\
\hline Gu et $\mathrm{a}^{43}$ & $\begin{array}{l}\text { Retrospective } \\
\text { cohort }\end{array}$ & $\begin{array}{l}\text { Healthcore } \\
\text { integrated } \\
\text { research } \\
\text { database }\end{array}$ & $\begin{array}{l}\text { RA patients }(\mathrm{N}=4,937) \\
\text { initiating first-line biologics } \\
\text { (new biologic users): ABA } \\
(\mathrm{n}=290), \text { ADA }(\mathrm{n}=\mathrm{I}, 47 \mathrm{I}), \\
\text { CER }(\mathrm{n}=\mid 32), \mathrm{ETN} \\
(\mathrm{n}=2,33 \mathrm{I}), \mathrm{GOL}(\mathrm{n}=\mid 84), \\
\text { IFX }(\mathrm{n}=529)\end{array}$ & $\begin{array}{l}\text { I year } \\
(2009-2013)\end{array}$ & $\begin{array}{l}\text { Persistent patients are those } \\
\text { with }<45 \text {-day gap in days of } \\
\text { supply on their index agent or } \\
\text { without a biologic switch }\end{array}$ & $\begin{array}{l}\text { ABA (45), ADA } \\
\text { (42), CER (33), } \\
\text { ETN (45), GOL } \\
\text { (34), IFX (47) }\end{array}$ \\
\hline $\begin{array}{l}\text { Howe } \\
\text { et } \mathrm{al}^{44}\end{array}$ & $\begin{array}{l}\text { Retrospective } \\
\text { cohort }\end{array}$ & $\begin{array}{l}\text { Humana } \\
\text { Commercial } \\
\text { Claims }\end{array}$ & $\begin{array}{l}\text { RA patients }(N=1,445 ; \\
\text { new/experienced biologic } \\
\text { users); new biologic users: } \\
\text { ABA }(n=42), A D A(n=238) \text {, } \\
\text { ETN }(n=280), G O L(n=10) \text {, } \\
\text { RIT ( } n=26) \text {, IFX }(n=71) \text {; } \\
\text { biologic experienced: ABA } \\
(n=37), A D A(n=23 \mid) \text {, } \\
\text { ETN }(n=338), G O L(n=4), \\
\text { RIT }(n=12), I F X(n=\mid 45)\end{array}$ & $\begin{array}{l}\text { I year } \\
(2007-2012)\end{array}$ & $\begin{array}{l}\text { Persistent patients are those } \\
\text { who continued on index } \\
\text { biologics without a } \geq 45 \text {-day } \\
\text { gap in therapy until the end of } \\
\text { the follow-up period, without a } \\
\text { switch to another biologic }\end{array}$ & $\begin{array}{l}\text { New biologic } \\
\text { users: ABA } \\
(4 I), \text { ADA } \\
\text { (43), ETN (46), } \\
\text { GOL (20), } \\
\text { RIT (42), IFX } \\
\text { (4I); biologic } \\
\text { experienced: } \\
\text { ABA (60), ADA } \\
\text { (60), ETN (60), } \\
\text { GOL (25), RIT } \\
\text { (50), IFX (63) }\end{array}$ \\
\hline $\begin{array}{l}\text { Fisher } \\
\text { et } \mathrm{al}^{45}\end{array}$ & $\begin{array}{l}\text { Retrospective } \\
\text { cohort }\end{array}$ & HIRD & $\begin{array}{l}\text { RA patients }(N=4,985) \text {, } \\
\text { including patients who were } \\
\text { new/experienced users } \\
\text { of biologics; new biologic } \\
\text { users: ETN }(n=I, 595) \text {, } \\
\text { ADA ( } n=4 \mid 7) \text {, IFX ( } n=4 \mid 4) \text {; } \\
\text { biologic experienced: ETN } \\
(n=I, 203), \text { ADA ( } n=507) \text {, } \\
\text { IFX }(n=849)\end{array}$ & $\begin{array}{l}\text { I year } \\
(2007-201 I)\end{array}$ & $\begin{array}{l}\text { Persistent patients are those } \\
\text { who did not have I) a number } \\
\text { of days from index date to } \\
\text { date of the first occurrence of } \\
\text { a switch to another biologic } \\
\text { DMARD, 2) a gap in index } \\
\text { therapy of } 45 \text { days between } \\
\text { the end of the day of supply } \\
\text { of the previous prescription } \\
\text { and the next prescription } \\
\text { renewal for self-injected } \\
\text { agents, }\end{array}$ & $\begin{array}{l}\text { New biologic } \\
\text { users: ETN } \\
\text { (62), ADA } \\
\text { (66), IFX } \\
\text { (69); biologic } \\
\text { experienced: } \\
\text { ETN (89), ADA } \\
\text { (94), IFX (96) }\end{array}$ \\
\hline
\end{tabular}

(Continued) 
Table 2 (Continued)

\begin{tabular}{|c|c|c|c|c|c|c|}
\hline Study & $\begin{array}{l}\text { Type of } \\
\text { study }\end{array}$ & $\begin{array}{l}\text { Source of } \\
\text { patient sample }\end{array}$ & Patient population & $\begin{array}{l}\text { Time } \\
\text { period }\end{array}$ & Persistence definition & $\begin{array}{l}\text { Persistence } \\
\text { rates (\%) }\end{array}$ \\
\hline & & & & & $\begin{array}{l}\text { and 3) I0I days from the } \\
\text { previous infliximab infusion } \\
\text { (8-week dosing interval plus the } \\
\text { 45-day gap) }\end{array}$ & \\
\hline $\begin{array}{l}\text { Greenberg } \\
\text { et } \mathrm{al}^{46}\end{array}$ & $\begin{array}{l}\text { Retrospective } \\
\text { analysis from } \\
\text { a prospective } \\
\text { registry }\end{array}$ & Corrona registry & $\begin{array}{l}\text { Newly TNFi prescribed } \\
\text { ( } n=I, 475 \text { biologically naïe, } \\
n=616 \text { first-time switchers, } \\
n=15 \text { I second-time or more } \\
\text { switchers) RA patients } \\
(N=2,242) \text {; new biologic } \\
\text { users: ADA ( } n=460) \text {, ETN } \\
(n=480) \text {, IFX ( } n=535) \text {; first- } \\
\text { time switcher: ADA ( } n=3 \mid \mathrm{I}) \text {, } \\
\text { ETN }(n=139), \text { IFX }(n=166)\end{array}$ & $\begin{array}{l}\text { I year and } \\
2 \text { years } \\
(2002-2008)\end{array}$ & $\begin{array}{l}\text { Time from initiation to } \\
\text { discontinuation of the anti- } \\
\text { TNF or to last follow-up visit; } \\
\text { treatment persistence was } \\
\text { estimated using survival analysis } \\
\text { methods }\end{array}$ & $\begin{array}{l}\text { New users } \\
\text { year I/2: ADA } \\
(68 / 53), \text { ETN } \\
(72 / 53), \text { IFX } \\
\text { (76/63); first- } \\
\text { time switchers } \\
\text { year I/2: ADA } \\
(57 / 42), \text { ETN } \\
(60 / 41), \text { IFX } \\
(65 / 43)\end{array}$ \\
\hline $\begin{array}{l}\text { Tang } \\
\text { et } \mathrm{al}^{47}\end{array}$ & $\begin{array}{l}\text { Retrospective } \\
\text { cohort }\end{array}$ & $\begin{array}{l}\text { PharMetrics: } \\
\text { managed-care } \\
\text { integrated } \\
\text { medical and } \\
\text { pharmacy claims }\end{array}$ & $\begin{array}{l}\text { RA patients }(N=1,242) \\
\text { receiving biologic and MTX } \\
\text { combination treatment } \\
\text { with previously filled } \\
\text { prescriptions; biologic and } \\
\text { MTX: ADA }(n=145), \text { ETN } \\
(n=607), \text { IFX }(n=490)\end{array}$ & $\begin{array}{l}\text { I year } \\
(2000-2005)\end{array}$ & $\begin{array}{l}\text { Persistence was defined as } \\
\text { the number of days between } \\
\text { the first and last anti-TNF } \\
\text { treatment and was reported as a } \\
\text { percentage of the I-year period } \\
\text { after treatment initiation }\end{array}$ & $\begin{array}{l}\text { ADA (7I), ETN } \\
(73), \text { IFX (78) }\end{array}$ \\
\hline $\begin{array}{l}\text { Yazici et } \\
\mathrm{al}^{48}\end{array}$ & $\begin{array}{l}\text { Retrospective } \\
\text { cohort }\end{array}$ & $\begin{array}{l}\text { PharMetrics } \\
\text { managed-care } \\
\text { integrated } \\
\text { medical and } \\
\text { pharmacy claims }\end{array}$ & $\begin{array}{l}\text { RA patients }(N=9,074) \text { who } \\
\text { had previously initiated } \\
\text { biologic treatment with } \\
\text { health plan eligibility } \\
3 \text { months prior to and } \\
6 \text { months after index; } \\
\text { population in } 2000 \text { : ETN } \\
\text { ( } n=563), \text { ADA ( } n=0) \text {, IFX } \\
(n=127) ; \text { population in } 2002 \text { : } \\
\text { ETN }(n=447) \text {, ADA }(n=0) \text {, } \\
\text { IFX }(n=736) ; \text { population } \\
\text { in } 2004 \text { : ETN }(n=I, 326) \text {, } \\
\text { ADA }(n=666) \text {, IFX }(n=706)\end{array}$ & $\begin{array}{l}\text { NR }(2000- \\
2005)\end{array}$ & $\begin{array}{l}\text { Persistent patients are } \\
\text { considered persistent until a } \\
30 \text {-day treatment gap after } \\
\text { completion of prior subscription } \\
\text { or a switch in biologic prior }\end{array}$ & $\begin{array}{l}\text { In 2000: ETN } \\
\text { (50), ADA } \\
\text { (NA), IFX } \\
\text { (65); in 2002: } \\
\text { ETN (7), ADA } \\
\text { (NA), IFX (35); } \\
\text { in 2004: ETN } \\
\text { ( } 1), \text { ADA (5), } \\
\text { IFX (20) }\end{array}$ \\
\hline $\begin{array}{l}\text { Cannon } \\
\text { et } \mathrm{al}^{49}\end{array}$ & $\begin{array}{l}\text { Retrospective } \\
\text { analysis from } \\
\text { a prospective } \\
\text { registry }\end{array}$ & VARA registry & $\begin{array}{l}\text { RA-confirmed patients } \\
(\mathrm{N}=563) \text { on first TNFi } \\
\text { therapy after March } 17, \\
2003 \text {, and enrolled in the } \\
\text { VA for at least } 6 \text { months } \\
\text { prior to first TNFi } \\
\text { prescription: ADA }(n=204) \text {, } \\
\text { ETN }(n=290), \text { IFX }(n=69)\end{array}$ & $\begin{array}{l}\text { I year, } \\
5 \text { years }\end{array}$ & $\begin{array}{l}\text { The number of days from the } \\
\text { course start date to course end } \\
\text { date or the date of a switch to } \\
\text { an alternative TNFi, whichever } \\
\text { came first; treatment persistence } \\
\text { was estimated using survival } \\
\text { analysis methods }\end{array}$ & $\begin{array}{l}\text { I year: ADA } \\
(64), \text { ETN } \\
(56), \text { IFX (67); } \\
5 \text { years: ADA } \\
(25), \text { ETN (I8), } \\
\text { IFX (2I) }\end{array}$ \\
\hline $\begin{array}{l}\text { Markenson } \\
\text { et } \mathrm{al}^{50}\end{array}$ & $\begin{array}{l}\text { Retrospective } \\
\text { analysis from } \\
\text { a prospective } \\
\text { registry }\end{array}$ & $\begin{array}{l}\text { RADIUS I } \\
\text { registry }\end{array}$ & $\begin{array}{l}\text { Patients with first exposure } \\
\text { to TNFi during the study } \\
\text { ( } N=2,4 \mid 8 \text {, including patients } \\
\text { who were biologic naive and } \\
\text { patients previously treated } \\
\text { with a biologic agent); } \\
\text { course I: ETN }(n=694) \text {, IFX } \\
(n=I, 427), \text { ADA }(n=297) \text {; } \\
\text { course 2: ETN }(n=248) \text {, } \\
\text { IFX }(n=\mid 30), \text { ADA }(n=277)^{c}\end{array}$ & $\begin{array}{l}5 \text { years } \\
(200 \mathrm{I}-2003)\end{array}$ & $\begin{array}{l}\text { Time from initiation of } \mathrm{TNFi} \\
\text { to discontinuation; treatment } \\
\text { persistence was estimated using } \\
\text { survival analysis methods }\end{array}$ & $\begin{array}{l}\text { Course I rates } \\
\text { for years I-5: } \\
\text { ETN }(78,66 \text {, } \\
61,53,5 \text { I); IFX } \\
(76,65,58,5 I \text {, } \\
48) \text {; ADA (80, } \\
65,52,48) ;{ }^{\circ} \\
\text { course } 2 \text { rates } \\
\text { for years I-3: } \\
\text { ETN }(74,59 \text {, } \\
56), \text { IFX (77, } \\
59,50), \text { ADA } \\
(69,52,46)\end{array}$ \\
\hline
\end{tabular}

(Continued) 
Table 2 (Continued)

\begin{tabular}{|c|c|c|c|c|c|c|}
\hline Study & $\begin{array}{l}\text { Type of } \\
\text { study }\end{array}$ & $\begin{array}{l}\text { Source of } \\
\text { patient sample }\end{array}$ & Patient population & $\begin{array}{l}\text { Time } \\
\text { period }\end{array}$ & Persistence definition & $\begin{array}{l}\text { Persistence } \\
\text { rates (\%) }\end{array}$ \\
\hline $\begin{array}{l}\text { Agarwal } \\
\text { et } \mathrm{al}^{51}\end{array}$ & $\begin{array}{l}\text { Prospective } \\
\text { cohort }\end{array}$ & BRASS & $\begin{array}{l}\text { RA patients }(\mathrm{N}=503) \\
\text { without a previous specific } \\
\text { treatment protocol ( } \mathrm{ETN} \text {, } \\
\text { ADA, IFX) }\end{array}$ & 3.25 years & $\begin{array}{l}\text { Persistent patients are those } \\
\text { who did not discontinue the } \\
\text { TNFi they reported using and } \\
\text { remained on the same TNFi }\end{array}$ & Overall (58) \\
\hline $\begin{array}{l}\text { Stockl } \\
\text { et } \mathrm{al}^{33}\end{array}$ & $\begin{array}{l}\text { Prospective } \\
\text { cohort }\end{array}$ & $\begin{array}{l}\text { PBM DTM } \\
\text { program }\end{array}$ & $\begin{array}{l}\text { RA patients }(\mathrm{N}=5 \mathrm{I} \text { 8) on } \\
\text { injectable RA medications } \\
\text { (ETN, ADA, ANA, ABA, IFX, } \\
\text { RIT): Group I - study ITT } \\
\text { ( } \mathrm{n}=340) \text {; Group } 2 \text { - study } \\
\text { completer ( } \mathrm{n}=244) \text {; Group } 3 \\
\text { - specialty pharmacy ( } \mathrm{n}=244) \text {; } \\
\text { Group } 4 \text { - community } \\
\text { pharmacy }(\mathrm{n}=244)\end{array}$ & $\begin{array}{l}0.67 \text { year } \\
(2007-2008)\end{array}$ & $\begin{array}{l}\text { Persistent patients are those } \\
\text { without a 30-day gap between } \\
\text { depletion date (fill date plus } \\
\text { days' supply) for the last filled } \\
\text { prescription and the end of the } \\
\text { post-identification period or } \\
\text { switching from index injectable } \\
\text { RA medication to another } \\
\text { injectable RA medication }\end{array}$ & $\begin{array}{l}\text { Group I (77), } \\
\text { Group } 2 \text { (87), } \\
\text { Group } 3 \text { (73), } \\
\text { Group } 4 \text { (52) }\end{array}$ \\
\hline $\begin{array}{l}\text { Agarwal } \\
\text { et } \mathrm{al}^{52}\end{array}$ & $\begin{array}{l}\text { Retrospective } \\
\text { chart review }\end{array}$ & $\begin{array}{l}\text { Hospital infusion } \\
\text { center pharmacy } \\
\text { and EMRs }\end{array}$ & $\begin{array}{l}\text { RA patients }(\mathrm{N}=183) \\
\text { receiving at least one } \\
\text { infusion of IFX }\end{array}$ & $\begin{array}{l}5 \text { years } \\
(1999-2003)\end{array}$ & $\begin{array}{l}\text { Persistent patients are those } \\
\text { who did not discontinue } \\
\text { treatments }\end{array}$ & Overall (33) \\
\hline $\begin{array}{l}\text { Bonafede } \\
\text { et } \mathrm{al}^{34}\end{array}$ & $\begin{array}{l}\text { Retrospective } \\
\text { cohort }\end{array}$ & $\begin{array}{l}\text { Truven Health } \\
\text { MarketScan } \\
\text { Commercial } \\
\text { Claims and } \\
\text { Encounters }\end{array}$ & $\begin{array}{l}\text { RA patients }(\mathrm{N}=4,542): \text { ETN } \\
+\mathrm{MTX}(\mathrm{n}=3,724), M T X+ \\
\mathrm{HCQ}+\mathrm{SSZ}(\mathrm{n}=8 \mid 8)\end{array}$ & $\begin{array}{l}\text { I year } \\
(2009-2013)\end{array}$ & $\begin{array}{l}\text { Persistent patients are those } \\
\text { with no treatment gap of } \\
>45 \text { days for any drug and no } \\
\text { addition or switching to other } \\
\text { DMARDs }\end{array}$ & $\begin{array}{l}\text { ETN + MTX } \\
\text { (29); MTX + } \\
\text { HCQ + SSZ } \\
(23)\end{array}$ \\
\hline $\begin{array}{l}\text { Bolge } \\
\text { et } \mathrm{a}^{53}\end{array}$ & $\begin{array}{l}\text { Cross- } \\
\text { sectional }\end{array}$ & $\begin{array}{l}\text { National Health } \\
\text { and Wellness } \\
\text { Surveys and } \\
\text { the Light Speed } \\
\text { Research } \\
\text { Ailment Panel }\end{array}$ & $\begin{array}{l}\text { RA existing biologic users } \\
(\mathrm{N}=250)\end{array}$ & $\begin{array}{l}\text { NA (2008- } \\
2010)\end{array}$ & $\begin{array}{l}\text { Persistent patients are those } \\
\text { who did not discontinue } \\
\text { biologics in the } 12 \text {-month period }\end{array}$ & $\begin{array}{l}\text { Overall (5I) } \\
\text { ADA }(6 \mathrm{I}) \\
\text { CER }(90) \\
\text { ETN }(66) \\
\text { GOL }(92)\end{array}$ \\
\hline \multicolumn{7}{|c|}{ PsO studies } \\
\hline $\begin{array}{l}\text { Bonafede } \\
\text { et } \mathrm{al}^{54}\end{array}$ & $\begin{array}{l}\text { Retrospective } \\
\text { cohort }\end{array}$ & $\begin{array}{l}\text { Truven Health } \\
\text { Analytics } \\
\text { Market Scan } \\
\text { Commercial } \\
\text { Claims and } \\
\text { Encounters }\end{array}$ & $\begin{array}{l}\text { PsO patients ( } N=2775 \text {; new } / \\
\text { experienced biologic users): } \\
\text { ADA }(n=1,166) \text {, ETN } \\
(n=1,609)\end{array}$ & $\begin{array}{l}\text { I year } \\
(2005-2010)\end{array}$ & $\begin{array}{l}\text { Persistent patients are those } \\
\text { who remained on biologic and } \\
\text { did not switch or have a therapy } \\
\text { gap of } \geq 45 \text { days }\end{array}$ & $\begin{array}{l}\text { ADA (57), ETN } \\
(46)\end{array}$ \\
\hline $\begin{array}{l}\text { Sauer } \\
\text { et } \mathrm{a}^{42}\end{array}$ & $\begin{array}{l}\text { Retrospective } \\
\text { cohort }\end{array}$ & VHA & $\begin{array}{l}\text { PsO patients }(\mathrm{N}=3,426) \\
\text { with one biologic } \\
\text { prescription during study } \\
\text { period (new/experienced } \\
\text { biologic users); new biologic } \\
\text { users: ADA ( } n=924) \text {, ETN } \\
\text { ( } n=1,136) \text {, INF ( } n=20) \text {; } \\
\text { biologic experienced: ADA } \\
(n=260), \text { ETN }(n=1,047), \\
\text { INF }(n=16)\end{array}$ & $\begin{array}{l}\text { I year } \\
(2008-2011)\end{array}$ & $\begin{array}{l}\text { Persistent patients are those } \\
\text { who continued on index } \\
\text { biologics without a } \geq 45 \text {-day } \\
\text { gap in therapy until the end of } \\
\text { the follow-up period, without a } \\
\text { switch to another biologic }\end{array}$ & $\begin{array}{l}\text { New biologic } \\
\text { users: ADA } \\
\text { (5I), ETN } \\
\text { (47), INF } \\
\text { (55); biologic } \\
\text { experienced: } \\
\text { ADA (58), ETN } \\
\text { (50), INF (75) }\end{array}$ \\
\hline $\begin{array}{l}\text { Howe } \\
\text { et } \mathrm{al}^{44}\end{array}$ & $\begin{array}{l}\text { Retrospective } \\
\text { cohort }\end{array}$ & $\begin{array}{l}\text { Humana } \\
\text { Commercial } \\
\text { Claims }\end{array}$ & $\begin{array}{l}\text { PsO patients ( } N=576 ; n e w / \\
\text { experienced biologic users); } \\
\text { new biologic users: ADA } \\
(n=\mid 26) \text {, ETN ( } n=\mid 55) ; \\
\text { biologic experienced: ADA } \\
(n=69), \text { ETN }(n=2 \mid 4)\end{array}$ & $\begin{array}{l}\text { I year } \\
(2007-2012)\end{array}$ & $\begin{array}{l}\text { Persistent patients are those } \\
\text { who continued on index } \\
\text { biologics without a } \geq 45 \text {-day } \\
\text { gap in therapy until the end of } \\
\text { the follow-up period, without a } \\
\text { switch to another biologic }\end{array}$ & $\begin{array}{l}\text { New biologic } \\
\text { users: ADA } \\
\text { (50), ETN } \\
\text { (34); biologic } \\
\text { experienced: } \\
\text { ADA (62), } \\
\text { ETN (48) }\end{array}$ \\
\hline $\begin{array}{l}\text { Chastek } \\
\text { et } \mathrm{al}^{55}\end{array}$ & $\begin{array}{l}\text { Retrospective } \\
\text { cohort }\end{array}$ & $\begin{array}{l}\text { National US } \\
\text { health plan } \\
\text { Optumlnsight }\end{array}$ & $\begin{array}{l}\text { PsO patients ( } \mathrm{N}=827 \text {; new } \\
\text { users), } \geq \mathrm{I} \text { medical claim } \\
\text { with a diagnosis for } \mathrm{PsO}\end{array}$ & $\begin{array}{l}\text { I year } \\
(2008)\end{array}$ & $\begin{array}{l}\text { Persistent patients are those } \\
\text { with no gaps in therapy } \geq 60 \\
\text { days }\end{array}$ & $\begin{array}{l}\text { ETN (42), ADA } \\
(40)\end{array}$ \\
\hline
\end{tabular}

(Continued) 
Table 2 (Continued)

\begin{tabular}{|c|c|c|c|c|c|c|}
\hline Study & $\begin{array}{l}\text { Type of } \\
\text { study }\end{array}$ & $\begin{array}{l}\text { Source of } \\
\text { patient sample }\end{array}$ & Patient population & $\begin{array}{l}\text { Time } \\
\text { period }\end{array}$ & Persistence definition & $\begin{array}{l}\text { Persistence } \\
\text { rates (\%) }\end{array}$ \\
\hline & & & $\begin{array}{l}\text { (ICD-9 code } 696.1) \text { from } \\
\text { baseline through } 30 \text { days } \\
\text { after the index date: ETN } \\
(n=330), \text { ADA }(n=497)\end{array}$ & & & \\
\hline $\begin{array}{l}\text { Feldman } \\
\text { et } \mathrm{al}^{56}\end{array}$ & $\begin{array}{l}\text { Retrospective } \\
\text { cohort }\end{array}$ & $\begin{array}{l}\text { MarketScan } \\
\text { Commercial } \\
\text { Encounters } \\
\text { Database }\end{array}$ & $\begin{array}{l}\mathrm{PsO} \text { patients }(\mathrm{N}=4,309) \\
\text { with } \geq 2 \text { claims for biologic } \\
\text { agents: ETN }(n=2,452) \\
\text { ADA }(n=1,662), \text { UST } \\
(n=195)\end{array}$ & $\begin{array}{l}\text { I year } \\
(2007-2012)\end{array}$ & $\begin{array}{l}\text { Persistent patients are those } \\
\text { with no treatment gaps } \\
\text { that exceeded } 4-18 \text { weeks, } \\
\text { dependent upon the biologic } \\
\text { prescribed }\end{array}$ & $\begin{array}{l}\text { ETN (I9), ADA } \\
\text { (53); UST (7I) }\end{array}$ \\
\hline Cao et $\mathrm{al}^{57}$ & $\begin{array}{l}\text { Retrospective } \\
\text { cohort }\end{array}$ & $\begin{array}{l}\text { Truven Health } \\
\text { MarketScan } \\
\text { Commercial } \\
\text { Claims and } \\
\text { Encounters }\end{array}$ & $\begin{array}{l}\text { PsO patients }(\mathrm{N}=\mathrm{I}, \mathrm{I52}) \text { with } \\
\geq \mathrm{I} \text { nondiagnostic medical } \\
\text { claim or prescription claim } \\
\text { indicating UST treatment: } \\
\text { UST }(\mathrm{n}=\mathrm{I}, 000)\end{array}$ & $\begin{array}{l}\text { I year } \\
(2009-2010)\end{array}$ & $\begin{array}{l}\text { Persistent patients are those } \\
\text { with continual usage over I } 30 \\
\text { days }\end{array}$ & UST (8I) \\
\hline \multicolumn{7}{|c|}{ PsA studies } \\
\hline $\begin{array}{l}\text { Shim } \\
\text { et } \mathrm{al}^{41}\end{array}$ & $\begin{array}{l}\text { Cross- } \\
\text { sectional }\end{array}$ & $\begin{array}{l}\text { Proprietary } \\
\text { administrative } \\
\text { claims database }\end{array}$ & $\begin{array}{l}\text { RA/PsA patients ( } N=I 5,834) \\
\text { with a biologic insurance } \\
\text { claim on TOC, CER, } \\
E T N, A D A \text {, and INF with } \\
\text { continuous enrollment in } \\
\text { health plan from index claim } \\
\text { to } 15 \text { months post-claim }\end{array}$ & $\begin{array}{l}\text { I year } \\
(2012-2013)\end{array}$ & $\begin{array}{l}\text { Persistent patients are those } \\
\text { with a treatment length of at } \\
\text { least } 365 \text { days after the index } \\
\text { claim without a therapy gap or } \\
\text { switch in therapy }\end{array}$ & Overall (67) \\
\hline $\begin{array}{l}\text { Sauer } \\
\text { et al }{ }^{42}\end{array}$ & $\begin{array}{l}\text { Retrospective } \\
\text { cohort }\end{array}$ & VHA & $\begin{array}{l}\text { PsA patients ( } N=875) \text { with } \\
\text { one biologic prescription } \\
\text { during study period (new/ } \\
\text { experienced biologic users); } \\
\text { new biologic users: ADA } \\
\text { ( } n=924) \text {, ETN ( } n=I, \mid 136) \text {, } \\
\text { INF ( } n=20) ; \text { biologic } \\
\text { experienced: ADA ( } n=260) \text {, } \\
\text { ETN }(n=I, 047), \text { INF }(n=16)\end{array}$ & $\begin{array}{l}\text { I year } \\
(2008-201 \mathrm{I})\end{array}$ & $\begin{array}{l}\text { Persistent patients are those } \\
\text { who continued on index } \\
\text { biologics without a } \geq 45 \text {-day } \\
\text { gap in therapy until the end of } \\
\text { the follow-up period, without a } \\
\text { switch to another biologic }\end{array}$ & $\begin{array}{l}\text { New biologic } \\
\text { users: ADA } \\
\text { (56), ETN } \\
\text { (5I), INF } \\
\text { (74); biologic } \\
\text { experienced: } \\
\text { ADA (56), ETN } \\
\text { (58), INF (79) }\end{array}$ \\
\hline $\begin{array}{l}\text { Mease } \\
\text { et } \mathrm{al}^{58}\end{array}$ & $\begin{array}{l}\text { Retrospective } \\
\text { analysis from } \\
\text { a prospective } \\
\text { registry }\end{array}$ & Corrona registry & $\begin{array}{l}\text { PsA patients }(\mathrm{N}=5 \mid 9 ; \\
\text { new users) with } 6 \text { months } \\
\text { post-follow-up after index } \\
\text { treatment: ADA }(n=2 \mid 4) \text {, } \\
\text { ETN }(n=\mid 55), I F X(n=\mid 10)\end{array}$ & $\begin{array}{l}\text { I year } \\
(2004-2012)\end{array}$ & $\begin{array}{l}\text { Median persistence: the length } \\
\text { of time that patients with PsA } \\
\text { maintained their initial biologic } \\
\text { therapy }\end{array}$ & $\begin{array}{l}\text { ADA: mono }(2.4 \\
\text { years), combo } \\
\text { ( } 2.4 \text { years); } \\
\text { ETN: mono ( } 3.9 \\
\text { years), combo } \\
\text { (I.6 years); } \\
\text { IFX: mono ( } 1.4 \\
\text { years), combo } \\
\text { (NR) }\end{array}$ \\
\hline $\begin{array}{l}\text { Chastek } \\
\text { et } \mathrm{al}^{59}\end{array}$ & $\begin{array}{l}\text { Retrospective } \\
\text { cohort }\end{array}$ & $\begin{array}{l}\text { Optumlnsight } \\
\text { Life Sciences } \\
\text { Research } \\
\text { Database }\end{array}$ & $\begin{array}{l}\text { PsA patients }(\mathrm{N}=346) \text { with } \\
\text { one previous claim and } \\
\text { continuously enrolled in } \\
\text { health plan: ADA ( } n=\mid 44) \text {, } \\
\text { ETN }(n=202)\end{array}$ & $\begin{array}{l}\text { ADA: } 1.2 \\
\text { years; ETN: } \\
\text { I year } \\
(2006-2008)\end{array}$ & $\begin{array}{l}\text { Persistent patients are those } \\
\text { with continuous use of index } \\
\text { medication without gaps in } \\
\text { therapy of at least } 60 \text { days }\end{array}$ & $\begin{array}{l}\text { ADA (50), ETN } \\
\text { (45) }\end{array}$ \\
\hline \multicolumn{7}{|c|}{ Mixed study ${ }^{\mathrm{e}}$} \\
\hline $\begin{array}{l}\text { Bonafede } \\
\text { et } \mathrm{al}^{54}\end{array}$ & $\begin{array}{l}\text { Retrospective } \\
\text { cohort }\end{array}$ & $\begin{array}{l}\text { Truven Health } \\
\text { Analytics Market- } \\
\text { Scan Commercial } \\
\text { Claims and } \\
\text { Encounters }\end{array}$ & $\begin{array}{l}\mathrm{PsO} / \mathrm{PsA}(\mathrm{N}=48 \mathrm{I}) \text { patients } \\
\text { who had initiated biologic } \\
\text { therapy: ADA }(\mathrm{n}=235) \text {, ETN } \\
(\mathrm{n}=246)\end{array}$ & $\begin{array}{l}\text { I year } \\
(2005-2010)\end{array}$ & $\begin{array}{l}\text { Persistent patients are those } \\
\text { who did not have to switch to } \\
\text { another TNFi or have a gap in } \\
\text { therapy of } \geq 45 \text { days over the } \\
\text { run-out of the fill }\end{array}$ & $\begin{array}{l}\text { PsA and PsO: } \\
\text { ADA (60), ETN } \\
(58)\end{array}$ \\
\hline
\end{tabular}

Notes: Median months of persistence. 'Percentage of days of being persistent. 'First-course therapy was defined as the first exposure to an anti-TNF agent at any time during the study. Second-course therapy was defined as initiation of any anti-TNF agent following the first discontinuation. ${ }^{d}$ Data for adalimumab was reported up to 4 years only. eData combined for multiple disease states (rather than separated by disease state).

Abbreviations: ABA, abatacept; ADA, adalimumab; ANA, anakinra; BRASS, Brigham RA Sequential Study; CER, certolizumab pegol; DMARD, disease-modifying antirheumatic drug; DTM, drug therapy management; EMR, electronic medical record; ETN, etanercept; GOL, golimumab; HCQ, hydroxychloroquine; HIRD, HealthCore Integrated Research Database; ICD-9, International Classification of Diseases, Ninth Revision; INF or IFX, infliximab; ITT, intent-to-treat; MAX, Medicaid Analytic Extract; MTX, methotrexate; NR, not reported; PBM, pharmacy benefits management; PsA, psoriatic arthritis; PsO, psoriasis; RA, rheumatoid arthritis; RADIUS, Rheumatoid Arthritis Disease-Modifying Anti-Rheumatic Drug Intervention and Utilization Study; RIT, rituximab; SSZ, sulfasalazine; TNFi, tumor necrosis factor alpha inhibitor; TOC, tocilizumab; US, United States; UST, ustekinumab; VA, Veterans Affairs; VARA, Veterans Affairs Rheumatoid Arthritis; VHA, Veterans Health Administration. 
being compared were not relevant because a majority of the studies were noncomparative in nature. A majority of the studies were considered to be of moderate to high quality for the domains that were applicable (Table 3 ).
Rates and factors associated with adherence

A total of 20 studies evaluated adherence rates in RA (14 studies), ${ }^{21-34} \mathrm{PsO}$ (six studies), ${ }^{35-40}$ or PsA (one study) ${ }^{32}$

Table 3 Risk of bias assessment ${ }^{a}$

\begin{tabular}{|c|c|c|c|c|c|c|c|c|c|c|c|c|}
\hline Author, year & I & 2 & 3 & 4 & 5 & 6 & 7 & 8 & 9 & 10 & I I & 12 \\
\hline Chu et al, ${ }^{21} 2015$ & Low & Low & Low & Low & Low & Low & Low & Low & Low & High & Low & High \\
\hline Erhardt et al, 222015 & Low & Low & Low & Low & NA & Low & Low & NA & Low & High & Low & Low \\
\hline Oladapo et al, ${ }^{23} 2014$ & Low & Low & Low & Low & Low & Low & Low & Low & Low & High & Low & Low \\
\hline Tkacz et al, ${ }^{24} 2014$ & Low & Low & Low & Low & Low & Low & Low & Low & Low & High & Low & Unclear \\
\hline Li et al, ${ }^{38} 2014$ & Low & Low & Low & Low & Low & Low & Low & Low & Low & Low & Unclear & Low \\
\hline Borah et al, ${ }^{26} 2009$ & Low & Low & Low & Low & Low & Low & Low & Low & Low & High & Low & Low \\
\hline Grijalva et al, ${ }^{27} 2010$ & Low & Low & Low & Low & Low & Low & Low & Low & Low & Low & Low & Low \\
\hline Harley et al, ${ }^{28} 2003$ & Low & Low & Low & Low & Low & Low & Low & High & Low & Unclear & Low & Unclear \\
\hline Curkendall et al,,92 2008 & Low & Low & Low & Low & Low & Low & Low & Low & Low & High & Low & High \\
\hline Grijalva et al, ${ }^{30} 2007$ & Low & Low & Low & Low & Low & Low & Low & Low & Low & High & Low & Unclear \\
\hline Barlow et al, ${ }^{31} 2012$ & Low & Low & Low & Low & Low & Low & Low & Unclear & Low & High & Low & High \\
\hline Ellis et al, ${ }^{32} 2014$ & Low & Low & Low & Low & Low & Low & Low & Low & Low & High & Low & High \\
\hline Stockl et al, ${ }^{33} 2010$ & Low & Low & Low & Low & Low & Low & Low & Unclear & Low & High & Low & Unclear \\
\hline Bonafede et al, ${ }^{54} 2013$ & Low & Low & Low & Low & Low & Low & Low & Low & Low & Low & Low & Low \\
\hline Sandoval et al, ${ }^{35} 2013$ & Low & Low & Low & Low & Unclear & Low & Low & Low & Low & High & Low & Unclear \\
\hline Doshi et al, ${ }^{36} 2015$ & Low & Low & Low & Low & Unclear & Low & Low & Low & Low & No & Low & Unclear \\
\hline Bhosle et al, ${ }^{37} 2006$ & NA & NA & NA & Low & NA & Low & NA & Low & Low & Low & Low & Low \\
\hline Li et al, ${ }^{25} 2010$ & Low & Low & Low & Low & Low & Low & Low & Low & Low & High & Low & Low \\
\hline Goren et al, ${ }^{39} 2016$ & Low & Low & Low & Low & Low & Low & Low & Low & Low & Low & Low & Low \\
\hline Kamangar et al, ${ }^{40} 2013$ & NA & NA & NA & Low & NA & Low & NA & Low & Low & Low & Unclear & Low \\
\hline Shim et al, ${ }^{41} 2015$ & Low & Low & Low & Low & Low & Low & Low & Low & Low & Low & Low & High \\
\hline Sauer et al, ${ }^{42} 2016$ & Low & Low & Low & Low & Low & Low & Low & Low & Low & High & Low & High \\
\hline Gu et al, ${ }^{43} 2015$ & Low & Low & Low & Low & Low & Low & Low & Low & High & High & Low & High \\
\hline Howe et al, ${ }^{44} 2014$ & Low & Low & Low & Low & Low & Low & Low & Low & Low & High & Low & Unclear \\
\hline Fisher et al, ${ }^{45} 2013$ & Low & Low & Low & Low & Low & Low & Low & Low & Low & High & Low & High \\
\hline Greenberg et al, ${ }^{46} 2012$ & Low & Low & Low & Low & Low & Low & Low & Low & Low & Low & Low & Low \\
\hline Tang et al, ${ }^{47} 2008$ & Low & Low & Low & Low & Low & Low & Low & High & Low & High & Low & Unclear \\
\hline Yazici et al, ${ }^{48} 2009$ & Low & Low & Low & Low & Low & Low & Low & Low & Low & High & Low & Low \\
\hline Cannon et $\mathrm{al}^{49} 2014$ & Low & Low & Low & Low & Low & Low & Low & Unclear & Low & High & Low & Unclear \\
\hline Markenson et al,,$^{50} 20 \mathrm{I} \mathrm{I}$ & Low & Low & Low & Low & Low & Low & Unclear & Unclear & Low & Low & Low & High \\
\hline Agarwal et al, ${ }^{51} 2008$ & Low & Low & Low & Low & Low & Low & Unclear & Low & Low & Low & Low & Low \\
\hline Bonafede et $a l,{ }^{34} 2015$ & Low & Low & Low & Low & Low & Low & Low & Low & Low & High & Low & High \\
\hline Agarwal et al, ${ }^{52} 2005$ & NA & NA & NA & Low & NA & Low & NA & Low & Low & Low & Low & Low \\
\hline Chastek et al, ${ }^{55} 2013$ & Low & Low & Low & Low & Low & Low & Low & Low & Low & Low & Low & Low \\
\hline Feldman et al, ${ }^{56} 2015$ & Low & Low & Low & Low & Low & Low & Low & Low & Low & High & Low & Low \\
\hline Cao et al, ${ }^{57} 2015$ & NA & NA & NA & Low & NA & Low & NA & Unclear & Low & Low & Low & High \\
\hline Mease et al, ${ }^{58} 2015$ & Low & Low & Low & Low & Unclear & Low & Low & Low & Low & Low & Unclear & Low \\
\hline Chastek et al, ${ }^{59} 2012$ & Low & Low & Low & Low & Low & Low & Low & Low & Low & Low & Low & Low \\
\hline Bolge et al,,$^{53} 2015$ & Low & Low & Low & Low & Low & Low & NA & Low & Low & Low & Low & Low \\
\hline Kwiatkowski et al, ${ }^{60} 2015$ & NA & NA & NA & Low & Low & Low & NA & Low & Unclear & Low & Low & Low \\
\hline Zhang et al,61 2013 & Low & Low & Low & Unclear & Low & Low & Low & Low & Low & Low & Low & High \\
\hline Menter et al, ${ }^{62} 2014$ & Low & Low & Low & Unclear & Low & Low & Low & Low & Low & Low & Low & Low \\
\hline Zhang et al, ${ }^{63} 2015$ & Low & Low & Low & Low & Low & Low & Low & Low & Low & Low & Low & Low \\
\hline
\end{tabular}

Notes: ${ }^{2}$ The table answers whether there is a low, high, or unclear risk of bias for these questions: I. Do the inclusion/exclusion criteria vary across the comparison groups of the study? 2. Does the strategy for recruiting participants into the study differ across groups? 3 . Is the selection of the comparison group inappropriate, after taking into account feasibility and ethical considerations? 4. Does the study fail to account for important variations in the execution of the study from the proposed protocol? 5. Was the outcome assessor not blinded to the intervention or exposure status of participants? 6 . Were valid and reliable measures implemented consistently across all study participants used to assess inclusion/exclusion criteria, intervention/exposure outcomes, participant health benefits and harms, and confounding? 7. Was the length of followup different across study groups? 8. In cases of high loss to follow-up (or differential loss to follow-up), was the impact assessed (eg, through sensitivity analysis or other adjustment method)? 9. Are any important primary outcomes missing from the results? I0. Are any important harms or adverse events that may be a consequence of the intervention/exposure missing from the results? II. Are results believable, taking study limitations into consideration? I2. Were important confounding variables not taken into account in the design and/or analysis (eg, through matching, stratification, interaction terms, multivariate analysis, or other statistical adjustment such as instrumental variables)?

Abbreviation: NA, not applicable. 
patient populations, with one study ${ }^{32}$ evaluating both RA and PsA patients (Table 1). Of these studies, nine analyzed data from private or commercial databases, six from government databases (Medicare/Medicaid/Veterans Affairs), two from registry data, and three from inpatient/outpatient hospital records (Table 1). When reported, follow-up periods were relatively consistent, ranging from 0.5 to 1 year. Individual studies measured adherence in various ways.

\section{Rheumatoid arthritis}

In RA, eight studies reported adherence using the standardized threshold of $\geq 80 \%$ for the PDC, the MPR, or the compliance ratio (number of therapy administrations or filled prescriptions divided by the expected number) methods over a 1-year time period. ${ }^{21-27,54}$ Adherence rates varied widely and were generally low. The median adherence rate for etanercept was 63\% (range, 16\%-73\%) and was reported in seven studies. ${ }^{21,23-26,28,34}$ The median adherence rate for adalimumab was also 63\% (range, 21\%-70\%) and was reported in four studies. ${ }^{23,24,26,27}$ Only three studies reported adherence rates for infliximab using the $\geq 80 \%$ MPR or PDC criteria $\left(38 \%, 43 \%\right.$, and $81 \%$ over 1 year). ${ }^{23,25,28}$ Although limited, in studies where multiple medications were studied simultaneously, adherence rates were greater for infliximab than for adalimumab or etanercept. ${ }^{23,28}$ For example, after matching for various baseline covariates, Oladapo et $\mathrm{al}^{23}$ reported a higher adherence rate for infliximab (38\%) users compared to adalimumab (21\%) and etanercept (16\%) users. Likewise, Harley et $\mathrm{al}^{28}$ reported a higher compliance ratio for infliximab users $(81 \%)$ than for etanercept $(68 \%)$ and methotrexate (64\%) users. Harley et $\mathrm{al}^{28}$ further corroborated the high adherence among infliximab users, reporting that etanercept (odds ratio [OR] 0.462; 95\% CI 0.290-0.736) and methotrexate (OR 0.385; 95\% CI 0.245-0.604) users were less likely to comply with their medications compared to infliximab users. Also using the criteria of $\geq 80 \%$ MPR or PDC, patients taking golimumab had a higher adherence rate $(81 \%$; $\mathrm{N}=261)$ than those taking adalimumab $(70 \% ; \mathrm{N}=1,532)$ or etanercept $(61 \% ; \mathrm{N}=2,099) .{ }^{24}$ Two studies assessed biologicmethotrexate combination therapy, and adherence rates were $26 \%$ and $28 \%$ over 1 year - higher than adherence to triple therapy combination of methotrexate, hydroxychloroquine, and sulfasalazine $(11 \%$ and $18 \%))^{22,54}$

Nine RA studies reported significant patient factors associated with better adherence using logistic regression analysis. ${ }^{21,24-26,28-30,54,60}$ Patient factors, including older age, ${ }^{21,24,25}$ male gender, ${ }^{24,29}$ other races (not including African Americans or Hispanics) compared with Whites, ${ }^{25}$ and
Whites compared with African Americans, ${ }^{21}$ were identified as more likely to be adherent.

Borah et $\mathrm{al}^{26}$ reported that experienced biologic users were more adherent than new users for both adalimumab and etanercept treatment groups (mean MPR, 70\% vs 63\% and $73 \%$ vs $65 \%$, respectively) and that experienced adalimumab users had $25 \%$ higher odds of nonadherence than experienced etanercept users (OR 1.25; 95\% CI 1.05-1.49). In another study, higher adherence (monotherapy: mean MPR) was observed for biologic monotherapies (90\% infliximab, $83 \%$ etanercept, $85 \%$ adalimumab) compared to biologicmethotrexate combination therapies (all mean MPRs $<75 \%$ ) or with methotrexate monotherapy $(80 \%){ }^{30}$

Another study found that patients with RA taking biologics who filled prescriptions through specialty pharmacies had higher adherence than those receiving medication from retail pharmacies. ${ }^{31}$ Over 3 years $(2006,2007$, and 2008), the mean adjusted adherence rates for specialty pharmacy and retail pharmacies patients were $63 \%$ vs $50 \%, 68 \%$ vs $51 \%$, and $61 \%$ vs $44 \%$, respectively.

\section{Psoriasis}

Six studies reported adherence rates on PsO (Table 1). ${ }^{35-40}$ Ustekinumab is the most frequently studied. Defining adherence as a $\mathrm{PDC} \geq 80 \%$ over 1 year among 2,707 patients with $\mathrm{PsO}$, Doshi et $\mathrm{al}^{36}$ found adherence to be the greatest with infliximab (49\%) users, followed by ustekinumab (43\%), adalimumab (41\%), and etanercept (29\%) users. Each of the other studies defined adherence differently (eg, never missing medication or injection, frequency of a missed dose, or adherence to recommended dosing schedule) and had fewer patient samples. Consequently, a wide range of adherence rates was observed (Table 1).

Three PsO studies assessed factors and reasons associated with medication nonadherence. ${ }^{37-39}$ A higher Charlson Comorbidity Index score is associated with decreased biologic adherence. ${ }^{37}$ In addition, being adherent to other prescribed $\mathrm{PsO}$ medications, including topical, phototherapy, or systemic drugs such as methotrexate, prednisone, acitretin, cyclosporine, and isotretinoin, was associated with better adherence to biologics. ${ }^{37}$ Older age, male gender, concomitant therapy, and low comorbid anxiety were associated with better adherence pattern. ${ }^{38}$ Based on a web-based survey, common reasons for nonadherence included the need to reschedule existing injection appointments $(7.4 \%$ adalimumab; $3.2 \%$ ustekinumab), the unaffordability of therapies (18.5\% adalimumab; $22.6 \%$ ustekinumab), forgetfulness (44.4\% adalimumab; 3.2\% ustekinumab), and other 
medical problems (not defined; 25.9\% adalimumab; 16.1\% ustekinumab). ${ }^{39}$

\section{Psoriatic arthritis}

Using the Truven Health MarketScan Commercial Claims database and defining medication adherence as proportion of adherent refills, one retrospective study of 325 patients with PsA reported a $76 \%$ adherence rate for golimumab users (Table 1). ${ }^{32}$

\section{Rates and factors associated with persistence}

A total of 23 studies evaluated medication persistence rates in RA (17 studies), PsO (six studies), and PsA (four studies) patient populations, with four studies ${ }^{41,42,44,54}$ evaluating more than one disease state for their patient population. Persistence was defined variably across studies (Table 2).

\section{Rheumatoid arthritis}

In the studies identified, persistence was usually measured as the time that patients continued treatment without a gap, discontinuation, or switching to another therapy over the specified time period. In most studies, the follow-up duration was 1 year and the gaps were $\geq 30,45$, or 90 days.

Similar to studies of adherence, a wide variation in persistence rates was observed, and the rates were generally low. However, unlike studies examining adherence, no biologic stood out as having higher persistence rates. Using the criteria of a gap of $\geq 45$ days over a 1 -year time period, the median persistence rate was $61 \%$ (range, 46\%-89\%) from four studies that evaluated etanercept. ${ }^{42-45}$ For adalimumab and infliximab, the median rates were $57.5 \%$ (range, $42 \%-94 \%$ ) and $63 \%$ (range, 41\%-76\%), respectively. ${ }^{42-45}$ Abatacept and golimumab rates were reported in three studies, ${ }^{42-44}$ with median rates of $52 \%$ (range, $41 \%-62 \%$ ) and $29.5 \%$ (range, $20 \%-55 \%$ ), respectively. Rates were $40 \%$ (range, 29\%-50\%) for rituximab ${ }^{42,44}$ and $33 \%$ for certolizumab. ${ }^{43}$

Three studies that used the same criteria ( $\geq 45$-day gap over a 1-year time period) presented the persistence rates by new or experienced biologic users, reporting consistently higher rates for experienced biologic users. ${ }^{42,44,45}$ However, one study of etanercept, adalimumab, and infliximab users over a 1- and 2-year time period reported that first-time switchers (nonestablished users) and second-time switchers had lower persistence rates, corroborated by higher likelihood of drug discontinuation (OR 1.42; 95\% CI 1.22-1.67; $P<0.001$ and OR 1.35; 95\% CI 1.03-1.76; $P=0.028$, respectively) compared to new biologic users. ${ }^{46}$ Several studies have compared biologic-methotrexate combinations with various conventional disease-modifying antirheumatic drug (cDMARD) regimens (eg, triple therapy combination of methotrexate, hydroxychloroquine, and sulfasalazine). Generally, persistence in the biologic combination groups was higher than in cDMARD combination patients. ${ }^{22}$ In comparing various biologic-methotrexate combinations, the mean persistence rate in the overall population over 1 year was $74.6 \%$, with higher rates for infliximab combination $(78 \%)$ compared to etanercept $(72.8 \%)$ and adalimumab $(70.8 \%)$ combination. ${ }^{47}$ Grijalva et $\mathrm{al}^{30}$ also reported that episodes of adalimumab-methotrexate combinations had a higher likelihood of persistence compared to methotrexate-only episodes (hazard ratio [HR] 0.63; 95\% CI 0.48-0.84).

As reported in three studies, patients taking infliximab tended to be more persistent compared to those taking etanercept or adalimumab, ${ }^{45,46,48}$ and another two studies reported that patients taking etanercept were likely more persistent than those taking adalimumab. ${ }^{26,30}$ However, there are notable exceptions where adalimumab-methotrexate combinations have greater persistence than infliximab-methotrexate or etanercept-methotrexate combinations, ${ }^{30}$ with some studies finding no differences in 1- or 5-year persistence rates between tumor necrosis factor (TNF) inhibitors. ${ }^{25,42,44,49,50}$

Several modifiable and nonmodifiable factors have been associated with persistence either positively or negatively among patients with RA. Nonmodifiable factors identified include age, gender, and race, whereas potentially modifiable factors identified include disease activity, comorbidities, out-of-pocket costs, combination therapy with methotrexate, cumulative use of methotrexate, and therapy management programs (disease therapy management and specialty pharmacy). ${ }^{29,30,46,50,51,61}$ Older patients tended to be persistent to infliximab (HR 0.99; 95\% CI 0.98-1.00; $P=0.002$ ); female patients (HR 1.24; 95\% CI 1.01-1.51; $P=0.040$ ) were more likely to discontinue etanercept; and White patients were more persistent than other races on etanercept. ${ }^{50}$ Markenson et $\mathrm{al}^{50}$ and Agarwal et $\mathrm{ll}^{51}$ reported that patients with higher disease activity (Clinical Disease Activity Index $>22$ : HR 1.35; 95\% CI 1.08-1.69; $P=0.009$; and RA Disease Activity Index scores: HR 1.13; 95\% CI 1.05-1.22) and physician global assessment scores (HR 1.27; 95\% CI 1.18-1.38) are more likely to discontinue TNF inhibitor use. Higher Charlson Comorbidity Index scores (HR 1.07; $P=0.002$ ) and higher weekly out-of-pocket costs $(>\$ 50)(\mathrm{HR} 1.58$; $P<0.001)$ increased the likelihood of nonpersistence to biologic therapy. ${ }^{29}$ In addition, cumulative use of methotrexate was associated with a decreased risk of TNF inhibitor 
discontinuation, as combined use of biologic and methotrexate was associated with a decreased risk of discontinuation compared to biologic monotherapy. ${ }^{30,46,51,61}$

One prospective study evaluated medication persistence to injectable RA medications (etanercept, adalimumab, anakinra, abatacept, infliximab, and rituximab) for patients participating in a disease therapy management program compared to nonparticipants receiving the same medication from specialty or community pharmacies over 0.67 years. ${ }^{33}$ The persistence rate ( $>30$-day gap or switching) was $77 \%$ for disease therapy management intent-to-treat patients, $86.8 \%$ for disease therapy management completers, $73 \%$ for specialty pharmacy patients, and $52 \%$ for community pharmacy patients. ${ }^{33}$

Other factors reported as reasons for low persistence were lack of effectiveness, continued disease activity, toxicity, and health insurance coverage/cost. ${ }^{52,53}$

\section{Psoriasis}

Six studies reported persistence patterns of biologics over a 1-year time period in $\mathrm{PsO} .^{34,42,44,55-57}$ Three of these use $\mathrm{a} \geq 45$-day gap period. Median persistence rates for etanercept and adalimumab were 47\% (range, 34\%-50\%) and $57 \%$ (range, $50 \%-62 \%$ ), respectively. ${ }^{42,44}$ Similar rates are observed when the definition was based on a $\geq 60$-day gap. ${ }^{55}$ In a retrospective study of MarketScan data, ustekinumab persistence $(71 \%)$ was higher than that for adalimumab (53\%) or etanercept (19\%); however, it should be noted that this study utilizes a gap criteria that depend on the biologic prescribed (a gap definition of $\geq 30$ days to $\geq 130$ days). ${ }^{56}$ In a separate study, ustekinumab persistence was high (81\%), with a gap definition of $\geq 130$ days. ${ }^{57}$

Clinical variables such as experience with drug and dosing may impact persistence. ${ }^{42,57}$ One study reported persistence for infliximab users as 55\% in new users and $75 \%$ in experienced users. ${ }^{42}$ Patients who initiated on the $90 \mathrm{mg}$ dose of ustekinumab showed lower persistence compared to those who initiated on the $45 \mathrm{mg}$ dose.${ }^{57}$ Reasons for stop/switch are similar across biologics, with lack of efficacy being the most frequent reason for nonpersistence.

\section{Psoriatic arthritis}

Four studies evaluated persistence rates in patients with PsA. ${ }^{41,42,58,59}$ Overall, persistence for all biologics, described as no treatment gap or switching over 1 year of treatment, was $61 \%$. Using a $\geq 45$-day gap criterion, persistence rates for etanercept, adalimumab, and infliximab were $51 \%, 56 \%$, and $74 \%$, respectively, in new users and $58 \%, 56 \%$, and $79 \%$, respectively, in established users. ${ }^{42}$ Similar rates for adalimumab and etanercept users were observed in another study using $\geq 60$-day gap criteria to describe persistence. ${ }^{41}$ Median duration of persistence with monotherapy and biologicmethotrexate combination therapies was 2.4 and 2.4 years, respectively, for adalimumab; 3.9 and 1.6 years, respectively, for etanercept; and 1.4 years for infliximab monotherapy (no data reported for methotrexate-infliximab combination). ${ }^{58}$

Two studies reported factors associated with biologic persistence in PsA. ${ }^{58,62}$ Shorter persistence is associated with a history of prior treatment with methotrexate, a history of coronary artery disease, a higher body mass index, and worse scores on most measures of baseline disability and disease activity. ${ }^{58}$ Ustekinumab was associated with a lower HR of discontinuing treatment (switching or stopping) compared to infliximab, adalimumab, and etanercept. ${ }^{62}$

\section{Discussion}

Since the inception of biologic therapies for the treatment of RA, patients with the disease have experienced an increase in health-related quality of life, relief of symptoms, and decrease in disease progression; however, proper adherence is the key to these positive outcomes because a lack of consistency to any such treatment regimen puts patients at a higher risk for disease reoccurrence and treatment failure. ${ }^{2}$ Successful treatment with biologics depends on ensuring long-term medication adherence; however, adherence rates differ between types of biologics and treatment schedules. ${ }^{36,53}$ In turn, this has an impact on the overall treatment effect of the available therapies.

This systematic literature review included a wide range of US studies describing adherence and persistence to biologics for patients with RA, PsO, or PsA. Most studies assessed patients with RA, with fewer studies available on patients with $\mathrm{PsO}$ and PsA. Various measures of adherence were used. Most commonly, MPR and PDC were used for reporting adherence, with an $80 \%$ cutoff predominantly used as a measure of successful adherence to treatment. Persistence was also defined differently across the studies but was most commonly reported as time from initial start (fill or refill) to treatment gap of $\geq 30,45$, or 90 days or to discontinuation. Variation in measures of adherence and persistence contributed to a wide range of adherence and persistence rates observed across studies. Etanercept, adalimumab, and infliximab were the most commonly studied biologics in the RA literature, and ustekinumab was the most common in the PsO literature. There was a notable lack of adherence data for patients with PsA. 
Overall, adherence and persistence were variable and low across the three disease states. Limited data were available to support a comparison of adherence for the same biologics in $\mathrm{RA}$ as compared with $\mathrm{PsO}$, although some suggest a difference in adherence to the same biologics between disease states. The reasons for observed differences are unknown. Adherence and persistence rates of approximately $50 \%-60 \%$ across the disease states were observed for commonly used therapies such as etanercept, adalimumab, and infliximab, although the observed ranges varied widely. Reasons that may contribute to the wide variation in observed adherence and persistence rates include differences in study populations, source of data, drug administration preference, and approaches used to measure adherence and persistence across the studies. Therefore, comparisons of rates between different drugs can be justifiable when drawn from the same study utilizing a comparable patient population and approach. For example, a few studies reported that infliximab users in RA were more adherent compared to adalimumab or etanercept users. ${ }^{23,25,28,30}$ This might be because of the wider interval schedule and intravenous route of infliximab administration in an outpatient setting compared to self-administered subcutaneous injections for etanercept and adalimumab. In a qualitative survey of patients' preferences, patients with RA preferred intravenous routes of administration (34.5\%) to subcutaneous administration (25.3\%), whereas patients with PsA preferred subcutaneous injections (45\%) to intravenous infusions $(9.7 \%){ }^{60}$

Many factors were associated with nonadherence or nonpersistence. Although some are demographic or socioeconomic in nature, others were related to patients' choices or their understanding of treatment pathways. Nonadherence among patients with RA and $\mathrm{PsO}$ and nonpersistence among patients with RA were associated with younger age, female gender, non-White race, and refilling prescriptions at retail pharmacies. Interestingly, biologic-methotrexate combination therapy was associated with lower adherence but also with higher persistence among patients with RA when compared to biologic monotherapy. Increased persistence among patients with RA using biologic-methotrexate combination therapy was in agreement with a recent study by Zhang et al, ${ }^{63}$ which showed that biologic-methotrexate combination therapy users are $30 \%$ less likely to be nonpersistent compared to biologic users. Although experience with biologics and the type of therapy affected adherence among patients with RA, they were also notable factors affecting persistence among patients with $\mathrm{PsO}$. High out-of-pocket cost, greater disease activity, and a high number of comorbidities were also deterrents to adherence or persistence. Qualitative surveys revealed that patients were not persistent because of perceived ineffectiveness and safety/tolerability concerns. In a survey, $59 \%$ of patients with PsA were not receiving any therapy or were receiving only a topical therapy, with $46 \%$ of them believing that the therapies were worse than the disease. ${ }^{3}$

Strengths of this systematic literature review on adherence and persistence of biologic users in three chronic inflammatory diseases include the variety of data sources used to inform this study, the different patient populations/ study designs, and the summarization of observational studies representing real-world experiences. Based on the most current available evidence, consistently low adherence and persistence rates for biologics were observed in all three disease states studied. In addition, the qualitative surveys from patients provided insight into the areas of concerns from the patients' perspectives. Inherent limitations to this systematic literature review include inconsistency in methodology across published studies and varied dosing schedules of different biologic drugs. These factors limit the ability to pool results for a quantitative summary and complicate standardization and generalizability of the findings. Additionally, because the data summarized here are secondary data based on the available literature, biases inherent in the studies may be present.

\section{Conclusion}

Despite the efficacy that biologics have on the outcomes of $\mathrm{RA}, \mathrm{PsO}$, and PsA patients, adherence and persistence rates to these medications were low, presenting significant opportunity for improvement. Various factors that may influence medication adherence or persistence patterns - including lower disease severity/activity; fewer comorbidities; lower out-of-pocket costs; refilling at specialty pharmacies; participation in disease management programs; and awareness of drug effectiveness, safety, and tolerability - point to areas where targeted improvements could be focused. These data should serve to guide future research to identify and implement innovative approaches that measure and reduce nonadherence or nonpersistence and improve treatment outcomes in RA, PsO, and PsA patients.

\section{Acknowledgments}

We are grateful to Michael Friedman for providing thorough data integrity review of the manuscript. The study was funded by Eli Lilly and Company. Abstract and poster presented at AMCP Nexus 2016 (https://www.jmcp.org/doi/pdf/10.18553/ jmcp.2016.22.issue-10-a). 


\section{Disclosure}

MJM, WNM, CL, TM, RTB, and ABA are employees and stockholders of Eli Lilly and Company. VT, CB, NJ, and SC are employees of ICON plc, which received funding from Eli Lilly and Company to conduct the study. SRF is an employee of the Wake Forest University School of Medicine and has received research, speaking, and consulting support from AbbVie, Celgene, Janssen, Lilly, and Novartis. The authors report no other conflicts of interest in this work.

\section{References}

1. Devaux S, Castela A, Archier E, et al. Adherence to topical treatment in psoriasis: a systematic literature review. J Eur Acad Dermatol Venereol. 2012;26(Suppl 3):61-67.

2. Fidder HH, Singendonk MM, van derHave M, Oldenburg B, van Oijen MG. Low rates of adherence for tumor necrosis factor- $\alpha$ inhibitors in Crohn's disease and rheumatoid arthritis: results of a systematic review. World J Gastroenterol. 2013;19(27):4344-4350.

3. Helliwell P, Coates L, Chandran V, et al. Qualifying unmet needs and improving standards of care in psoriatic arthritis. Arthritis Care Res. 2014;66(12):1759-1766.

4. Rathbun AM, Reed GW, Harrold LR. The temporal relationship between depression and rheumatoid arthritis disease activity, treatment persistence and response: a systematic review. Rheumatology. 2013; 52(10):1785-1794.

5. Helmick CG, Felson DT, Lawrence RC, et al. Estimates of the prevalence of arthritis and other rheumatic conditions in the United States. Part I. Arthritis Rheum. 2008;58(1):15-25.

6. Vandever L. Rheumatoid arthritis by the numbers: facts, statistics, and you. Available from: http://www.healthline.com/health/rheumatoidarthritis/facts-statistics-infographic. Accessed April 19, 2017.

7. Arthritis Foundation. How common is psoriatic arthritis in people with psoriasis? Available from: http://blog.arthritis.org/psoriatic-arthritis/ psoriatic-arthritis-psoriasis/. Accessed April 19, 2017.

8. Rachakonda TD, Schupp CW, Armstrong AW. Psoriasis prevalence among adults in the United States. J Am Acad Dermatol. 2014; 70(3):512-516.

9. Cramer JA, Roy A, Burrell A, Fairchild CJ, Fuldeore MJ, Ollendorf DA, Wong PK. Medication compliance and persistence: terminology and definitions. Value Health. 2008;11(1):44-47.

10. Cramer J, Rosenheck R, Kirk G, Krol W, Krystal J; VA Naltrexone Study Group 425. Medication compliance feedback and monitoring in a clinical trial: predictors and outcomes. Value Health. 2003;6(5):566-573.

11. Haynes RB, Mcdonald H, Garg AX, Montague P. Interventions for helping patients to follow prescriptions for medications. Cochrane Database Syst Rev. 2002;2(2):7-9.

12. Haynes RB, Mcdonald HP, Garg AX. Helping patients follow prescribed treatment: clinical applications. JAMA. 2002;288(22):2880-2883.

13. Osterberg L, Blaschke T. Adherence to medication. NEngl J Med. 2005; 353(5):487-497.

14. Iuga AO, Mcguire MJ. Adherence and health care costs. Risk Manag Healthc Policy. 2014;7:35-44.

15. Blum MA, Koo D, Doshi JA. Measurement and rates of persistence with and adherence to biologics for rheumatoid arthritis: a systematic review. Clin Ther. 2011;33(7):901-913.

16. Harrold LR, Andrade SE. Medication adherence of patients with selected rheumatic conditions: a systematic review of the literature. Semin Arthritis Rheum. 2009;38(5):396-402.

17. Pasma A, van't Spijker A, Hazes JM, Busschbach JJ, Luime JJ. Factors associated with adherence to pharmaceutical treatment for rheumatoid arthritis patients: a systematic review. Semin Arthritis Rheum. 2013; 43(1):18-28.
18. Thorneloe RJ, Bundy C, Griffiths CE, Ashcroft DM, Cordingley L. Adherence to medication in patients with psoriasis: a systematic literature review. Br J Dermatol. 2013;168(1):20-31.

19. Vangeli E, Bakhshi S, Baker A, et al. A systematic review of factors associated with non-adherence to treatment for immune-mediated inflammatory diseases. Adv Ther. 2015;32(11):983-1028.

20. Viswanathan M, Berkman ND, Dryden DM, Hartling L. Assessing Risk of Bias and Confounding in Observational Studies of Interventions or Exposures: Further Development of the RTI Item Bank. Rockville, MD: Agency for Healthcare Research and Quality; 2013.

21. Chu LH, Kawatkar AA, Gabriel SE. Medication adherence and attrition to biologic treatment in rheumatoid arthritis patients. Clin Ther. 2015;37(3):660-666.

22. Erhardt D, Sauer B, Teng CC. Clinical practice experience in rheumatoid arthritis patients treated with triple therapy and methotrexate-tumor necrosis factor inhibition differs from that of randomized controlled trials. Arthritis Rheumatol. 2015;67(Suppl 10):3197.

23. Oladapo A, Barner JC, Lawson KA, Novak S, Rascati KL, Richards KM, Harrison DJ. Medication effectiveness with the use of tumor necrosis factor inhibitors among Texas Medicaid patients diagnosed with rheumatoid arthritis. J Manag Care Spec Pharm. 2014;20(7): 657-667.

24. Tkacz J, Ellis L, Bolge SC, Meyer R, Brady BL, Ruetsch C. Utilization and adherence patterns of subcutaneously administered anti-tumor necrosis factor treatment among rheumatoid arthritis patients. Clin Ther. 2014;36(5):737-747.

25. Li P, Blum MA, von Feldt J, Hennessy S, Doshi JA. Adherence, discontinuation, and switching of biologic therapies in medicaid enrollees with rheumatoid arthritis. Value Health. 2010;13(6):805-812.

26. Borah BJ, Huang X, Zarotsky V, Globe D. Trends in RA patients' adherence to subcutaneous anti-TNF therapies and costs. Curr Med Res Opin. 2009;25(6):1365-1377.

27. Grijalva CG, Kaltenbach L, Arbogast PG, Mitchel EF, Griffin MR. Adherence to disease-modifying antirheumatic drugs and the effects of exposure misclassification on the risk of hospital admission. Arthritis Care Res. 2010;62(5):730-734.

28. Harley CR, Frytak JR, Tandon N. Treatment compliance and dosage administration among rheumatoid arthritis patients receiving infliximab, etanercept, or methotrexate. Am J Manag Care. 2003; 9(6 Suppl):S136-S143.

29. Curkendall S, Patel V, Gleeson M, Campbell RS, Zagari M, Dubois R. Compliance with biologic therapies for rheumatoid arthritis: do patient out-of-pocket payments matter? Arthritis Rheum. 2008;59(10): 1519-1526.

30. Grijalva CG, Chung CP, Arbogast PG, Stein CM, Mitchel EF, Griffin MR. Assessment of adherence to and persistence on diseasemodifying antirheumatic drugs (DMARDs) in patients with rheumatoid arthritis. Med Care. 2007;45(10 Suppl 2):S66-S76.

31. Barlow JF, Faris RJ, Wang W, Verbrugge RR, Garavaglia SB, Aubert RE. Impact of specialty pharmacy on treatment costs for rheumatoid arthritis. Am J Pharm Benefits. 2012;4(6):SP49-SP56.

32. Ellis L, Bolge S, Rice P. Golimumab utilization patterns and refill adherence in patients with rheumatoid arthritis, psoriatic arthritis and ankylosing spondylitis. Value Health. 2014;17(3):A49-A50.

33. Stockl KM, Shin JS, Lew HC, Zakharyan A, Harada AS, Solow BK, Curtis BS. Outcomes of a rheumatoid arthritis disease therapy management program focusing on medication adherence. J Manag Care Pharm. 2010;16(8):593-604

34. Bonafede M, Johnson BH, Tang DH, Shah N, Harrison DJ, Collier DH. Etanercept-methotrexate combination therapy initiators have greater adherence and persistence than triple therapy initiators with rheumatoid arthritis. Arthritis Care Res. 2015;67(12):1656-1663.

35. Sandoval LF, Huang KE, Feldman SR. Adherence to ustekinumab in psoriasis patients. J Drugs Dermatol. 2013;12(10):1090-1092. 
36. Doshi JA, Takeshita J, Pinto L, et al. Biologic adherence among psoriasis patients in the US Medicare population. Value Health. 2015; 18(3):A186.

37. Bhosle MJ, Feldman SR, Camacho FT, Timothy Whitmire J, Nahata MC, Balkrishnan R. Medication adherence and health care costs associated with biologics in Medicaid-enrolled patients with psoriasis. J Dermatolog Treat. 2006;17(5):294-301.

38. Li Y, Zhou H, Cai B, Kahler KH, Tian H, Gabriel S, Arcona S. Groupbased trajectory modeling to assess adherence to biologics among patients with psoriasis. Clinicoecon Outcomes Res. 2014;6:197-208.

39. Goren A, Carter C, Lee S. Patient reported health outcomes and non-adherence in psoriasis patients receiving adalimumab or ustekinumab for moderate to severe plaque psoriasis. $J$ Dermatolog Treat. 2016;27(1):43-50

40. Kamangar F, Isip L, Bhutani T, et al. How psoriasis patients perceive, obtain, and use biologic agents: survey from an academic medical center. J Dermatolog Treat. 2013;24(1):13-24.

41. Shim A, Pham H, Fairman K. Association of medication persistency with route of administration and patient cost-sharing: analysis of commonly used biologics. Value Health. 2015;18(7):A650.

42. Sauer BC, Teng CC, He T, et al. Treatment patterns and annual biologic costs in US veterans with rheumatic conditions or psoriasis. J Med Econ. 2016;19(1):34-43.

43. Gu T, Shah N, Deshpande G, Tang DH, Eisenberg D, Harrison DJ. Persistence with first-line biologics used in rheumatoid arthritis in a US managed care population. Value Health. 2015;18(3):A168.

44. Howe A, Eyck LT, Dufour R, Shah N, Harrison DJ. Treatment patterns and annual drug costs of biologic therapies across indications from the Humana commercial database. J Manag Care Spec Pharm. 2014;20(12):1236-1244.

45. Fisher MD, Watson C, Fox KM, Chen YW, Gandra SR. Dosing patterns of three tumor necrosis factor blockers among patients with rheumatoid arthritis in a large United States managed care population. Curr Med Res Opin. 2013;29(5):561-568.

46. Greenberg JD, Reed G, Decktor D, et al. A comparative effectiveness study of adalimumab, etanercept and infliximab in biologically naive and switched rheumatoid arthritis patients: results from the US CORRONA registry. Ann Rheum Dis. 2012;71(7):1134-1142.

47. Tang B, Rahman M, Waters HC, Callegari P. Treatment persistence with adalimumab, etanercept, or infliximab in combination with methotrexate and the effects on health care costs in patients with rheumatoid arthritis. Clin Ther. 2008;30(7):1375-1384.

48. Yazici Y, Krasnokutsky S, Barnes JP, Hines PL, Wang J, Rosenblatt L. Changing patterns of tumor necrosis factor inhibitor use in 9074 patients with rheumatoid arthritis. J Rheumatol. 2009;36(5):907-913.

49. Cannon GW, Duvall SL, Haroldsen CL, et al. Persistence and dose escalation of tumor necrosis factor inhibitors in US veterans with rheumatoid arthritis. J Rheumatol. 2014;41(10):1935-1943.
50. Markenson JA, Gibofsky A, Palmer WR, Keystone EC, Schiff MH, Feng J, Baumgartner SW. Persistence with anti-tumor necrosis factor therapies in patients with rheumatoid arthritis: observations from the RADIUS registry. J Rheumatol. 2011;38(7):1273-1281.

51. Agarwal SK, Glass RJ, Shadick NA, et al. Predictors of discontinuation of tumor necrosis factor inhibitors in patients with rheumatoid arthritis. J Rheumatol. 2008;35(9):1737-1744.

52. Agarwal SK, Maier AL, Chibnik LB, et al. Pattern of infliximab utilization in rheumatoid arthritis patients at an academic medical center. Arthritis Rheum. 2005;53(6):872-878.

53. Bolge SC, Goren A, Tandon N. Reasons for discontinuation of subcutaneous biologic therapy in the treatment of rheumatoid arthritis: a patient perspective. Patient Prefer Adherence. 2015;9:121-131.

54. Bonafede M, Johnson BH, Fox KM, Watson C, Gandra SR. Treatment patterns with etanercept and adalimumab for psoriatic diseases in a real-world setting. J Dermatolog Treat. 2013;24(5):369-373.

55. Chastek B, Fox KM, Watson C, Kricorian G, Gandra SR. Psoriasis treatment patterns with etanercept and adalimumab in a United States health plan population. J Dermatolog Treat. 2013;24(1):25-33.

56. Feldman SR, Zhao Y, Navaratnam P, Friedman HS, Lu J, Tran MH. Patterns of medication utilization and costs associated with the use of etanercept, adalimumab, and ustekinumab in the management of moderate-to-severe psoriasis. J Manag Care Spec Pharm. 2015;21(3): 201-209.

57. Cao Z, Carter C, Wilson KL, Schenkel B, Dosing U. Ustekinumab dosing, persistence, and discontinuation patterns in patients with moderateto-severe psoriasis. J Dermatolog Treat. 2015;26(2):113-120.

58. Mease PJ, Collier DH, Saunders KC, Li G, Kremer JM, Greenberg JD. Comparative effectiveness of biologic monotherapy versus combination therapy for patients with psoriatic arthritis: results from the Corrona registry. RMD Open. 2015;1(1):e000181.

59. Chastek B, Fox KM, Watson C, Gandra SR. Etanercept and adalimumab treatment patterns in psoriatic arthritis patients enrolled in a commercial health plan. Adv Ther. 2012;29(8):691-697.

60. Kwiatkowski A, Grisanti L, Grisanti J, Hatem J. Patient preferences regarding route of biologic administration in an inflammatory arthritis cohort. Arthritis Rheumatol. 2015;67(Suppl 10):2326.

61. Zhang J, Xie F, Delzell ES, et al. Persistence on biologics is associated with concomitant methotrexate use among rheumatoid arthritis patient. Arthritis Rheum. 2013;65:S990-S991.

62. Menter A, Papp K, Krueger GG, et al. Persistence of biologic therapy in psoriatic disease: results from the psoriasis longitudinal assessment and registry. Arthritis Rheum. 2014;66:S693.

63. Zhang J, Xie F, Delzell E, et al. Impact of biologic agents with and without concomitant methotrexate and at reduced doses in older rheumatoid arthritis patients. Arthritis Care Res. 2015;67(5):624-632. 


\section{Supplementary materials}

Search strategy.

Embase 1974 to 2015 December 28, 2015 using OvidSp

\begin{tabular}{|c|c|c|}
\hline I & *rheumatoid arthritis/ & 97,740 \\
\hline 2 & *rheumatoid nodule/ & 651 \\
\hline 3 & (rheumatoid adj nodule).ti,ab. & 295 \\
\hline 4 & (arthritis adj2 rheumat\$).ti,ab. & 119,688 \\
\hline 5 & RA.ti,ab. & 85,007 \\
\hline 6 & *psoriasis/ & 31,038 \\
\hline 7 & psoria\$.ti,ab. & 52,099 \\
\hline 8 & *psoriatic arthritis/ & 6,451 \\
\hline 9 & psoriatic arthrit\$.ti,ab. & 9,783 \\
\hline 10 & or/l-9 & 224,711 \\
\hline 11 & *medication compliance/ & 3,158 \\
\hline 12 & *patient compliance/ & 19,028 \\
\hline 13 & (cooperat $\$$ or co-operat\$).ti,ab. & 137,785 \\
\hline 14 & *treatment refusal/ & 3,763 \\
\hline 15 & (treatment adj2 refusal).ti,ab. & 652 \\
\hline 16 & *patient dropouts/ & 107 \\
\hline 17 & patient dropout\$.ti,ab. & 212 \\
\hline 18 & (uncooperat $\$$ or unco-operat $\$$ ).ti,ab. & $\mathrm{I}, 488$ \\
\hline 19 & (patient adj perspective\$).ti,ab. & 2,682 \\
\hline 20 & (patient adj attitude\$).ti,ab. & $\mathrm{I}, 047$ \\
\hline 21 & (patient adj experience \$).ti,ab. & 15,219 \\
\hline 22 & continuation rate $\$ . t i, a b$. & 1,162 \\
\hline 23 & (adher $\$$ adj5 (regimen $\$$ or medication $\$$ or treatment $\$$ or therap $\$$ or drug $\$$ or intervention $\$$ or biologic $\$$ )).ti,ab. & 41,915 \\
\hline 24 & (complian $\$$ adj5 (regimen $\$$ or patient $\$$ or medication $\$$ or treatment $\$$ or therap $\$$ or drug $\$$ or intervention $\$$ or biologic $\$$ )).ti,ab. & 50,669 \\
\hline 25 & (persist $\$$ adj 5 (regimen $\$$ or medication $\$$ or treatment $\$$ or therap $\$$ or drug $\$$ or intervention $\$$ or biologic $\$$ )).ti,ab. & 31,812 \\
\hline 26 & $\begin{array}{l}((\text { nonadher } \$ \text { or non-adher } \$ \text { or non adher } \$ \text { ) adj5 (regimen } \$ \text { or medication } \$ \text { or treatment } \$ \text { or therap } \$ \text { or drug } \$ \text { or } \\
\text { intervention } \$ \text { or biologic } \$) \text { ).ti,ab. }\end{array}$ & 6,231 \\
\hline 27 & $\begin{array}{l}((\text { nonpersist } \$ \text { or non-persist } \$ \text { or non persist } \$ \text { ) adj5 (regimen } \$ \text { or medication } \$ \text { or treatment } \$ \text { or therap } \$ \text { or drug } \$ \text { or } \\
\text { intervention } \$ \text { or biologic } \$)) \text {.ti,ab. }\end{array}$ & 233 \\
\hline 28 & $\begin{array}{l}((\text { noncomplian } \$ \text { or non-complian } \$ \text { or non complian } \$ \text { ) adj5 (patient } \$ \text { or regimen } \$ \text { or medication } \$ \text { or treatment } \$ \text { or therap } \$ \text { or } \\
\text { drug } \$ \text { or intervention } \$ \text { or biologic } \$) \text { ).ti,ab. }\end{array}$ & 7,993 \\
\hline 29 & (assessment adj5 (adher\$ or complian\$ or persist\$)).ti,ab. & 3,684 \\
\hline 30 & (assessment adj5 (nonadher $\$$ or non-adher $\$$ or non adher $\$$ )).ti,ab. & 85 \\
\hline 31 & (assessment adj5 (noncomplian\$ or non-complian\$ or non complian\$)).ti,ab. & 81 \\
\hline 32 & (assessment adj5 (nonpersist $\$$ or non-persist $\$$ or non persist $\$$ or nonpersist $\$$ )).ti,ab. & 5 \\
\hline 33 & or/l1-32 & 294,808 \\
\hline 34 & 10 and 33 & 3,614 \\
\hline 35 & $\begin{array}{l}\text { limit } 34 \text { to (conference abstract or conference paper or conference proceeding or "conference review" or editorial or letter } \\
\text { or "review") }\end{array}$ & 1,764 \\
\hline 36 & 34 not 35 & 1,850 \\
\hline 37 & limit 36 to human & 1,478 \\
\hline
\end{tabular}




\section{Ovid Medline (R) in-process and other non-indexed citations and Ovid and Medline (R) 1946-December 28, 20I5 using OvidSp}

\begin{tabular}{|c|c|c|}
\hline I & *Arthritis, Rheumatoid/ & 69,229 \\
\hline 2 & *Rheumatoid Nodule/ & 567 \\
\hline 3 & (rheumatoid adj nodule).ti,ab. & 240 \\
\hline 4 & (arthritis adj2 rheumat\$).ti,ab. & 86,167 \\
\hline 5 & RA.ti,ab. & 56,769 \\
\hline 6 & *Psoriasis/ & 23,577 \\
\hline 7 & psoria $\$ . t i, a b$. & 35,733 \\
\hline 8 & psoriatic arthrit\$.ti,ab. & 5,659 \\
\hline 9 & *Arthritis, Psoriatic/ & $3,|7|$ \\
\hline 10 & or/l-9 & 161,396 \\
\hline II & *Medication Adherence/ & 6,402 \\
\hline 12 & *Patient Compliance/ & 20,742 \\
\hline 13 & (adher $\$$ adj5 (regimen $\$$ or medication $\$$ or treatment $\$$ or therap $\$$ or drug $\$$ or intervention $\$$ or biologic $\$$ )).ti,ab. & 27,924 \\
\hline 14 & (complian $\$$ adj5 (regimen $\$$ or patient $\$$ or medication $\$$ or treatment $\$$ or therap $\$$ or drug $\$$ or intervention $\$$ or biologic $\$$ )).ti,ab. & 32,328 \\
\hline 15 & (persist $\$$ adj5 (regimen $\$$ or medication $\$$ or treatment $\$$ or therap $\$$ or drug $\$$ or intervention $\$$ or biologic $\$$ )).ti,ab. & 23,065 \\
\hline 16 & cooperat $\$$ or co-operat $\$) . t i, a b$. & 118,393 \\
\hline 17 & *Treatment Refusal/ & 5,526 \\
\hline 18 & (treatment adj2 refusal).ti,ab. & 514 \\
\hline 19 & *Patient Dropouts/ & 2,516 \\
\hline 20 & patient dropout $\$ . t i, a b$. & 172 \\
\hline 21 & $\begin{array}{l}((\text { nonadher } \$ \text { or non-adher } \$ \text { or non adher } \$ \text { ) adj5 (regimen } \$ \text { or medication } \$ \text { or treatment } \$ \text { or therap } \$ \text { or drug } \$ \text { or } \\
\text { intervention } \$ \text { or biologic } \$) \text { ).ti,ab. }\end{array}$ & 4,056 \\
\hline 22 & $\begin{array}{l}\text { ( (nonpersist } \$ \text { or non-persist } \$ \text { or non persist } \$ \text { ) adj5 (regimen } \$ \text { or medication } \$ \text { or treatment } \$ \text { or therap } \$ \text { or drug } \$ \text { or } \\
\text { intervention } \$ \text { or biologic } \$)) \text {.ti,ab. }\end{array}$ & $|3|$ \\
\hline 23 & $\begin{array}{l}((\text { noncomplian } \$ \text { or non-complian } \$ \text { or non complian } \$ \text { ) adj5 (patient } \$ \text { or regimen } \$ \text { or medication } \$ \text { or treatment } \$ \text { or therap } \$ \text { or } \\
\text { drug } \$ \text { or intervention } \$ \text { or biologic } \$) \text { ).ti,ab. }\end{array}$ & 5,319 \\
\hline 24 & (uncooperat $\$$ or unco-operat\$).ti,ab. & 1,188 \\
\hline 25 & (patient adj perspective\$).ti,ab. & $\mathrm{I}, 878$ \\
\hline 26 & (patient adj attitude\$).ti,ab. & 811 \\
\hline 27 & (patient adj experience $\$$ ).ti,ab. & 10,173 \\
\hline 28 & continuation rate $\$ . t i, a b$. & $\mathrm{I}, 142$ \\
\hline 29 & (assessment adj5 (adher $\$$ or complian $\$$ or persist $\$$ )).ti,ab. & 2,418 \\
\hline 30 & (assessment adj5 (nonadher $\$$ or non-adher $\$$ or non adher $\$$ )).ti,ab. & 57 \\
\hline 31 & (assessment adj5 (noncomplian $\$$ or non-complian $\$$ or non complian $\$$ )).ti,ab. & 49 \\
\hline 32 & (assessment adj5 (nonpersist $\$$ or non-persist $\$$ or non persist $\$$ or nonpersist $\$$ )).ti,ab. & 4 \\
\hline 33 & or/l1-32 & 235,799 \\
\hline 34 & 10 and 33 & 2,041 \\
\hline 35 & limit 34 to (case reports or comment or in vitro or lectures or letter or "review") & 467 \\
\hline 36 & 34 not 35 & 1,574 \\
\hline 37 & limit 36 to humans & $\mathrm{I}, 250$ \\
\hline
\end{tabular}




\section{Cochrane, December 28, 2015 (CENTRAL only)}

\begin{tabular}{lll}
\hline I & MeSH descriptor: [Arthritis, Rheumatoid] explode all trees & 4,206 \\
2 & MeSH descriptor: [Rheumatoid Nodule] explode all trees & II \\
3 & rheumatoid next nodule*ti,ab,kw (Word variations have been searched) & 17 \\
4 & arthritis near/3 rheumat*:ti,ab,kw (Word variations have been searched) & 7,762 \\
5 & RA:ti,ab,kw (Word variations have been searched) & 4,379 \\
6 & MeSH descriptor: [Psoriasis] explode all trees & 1,916 \\
7 & psoria*:ti,ab,kw (Word variations have been searched) & 4,176 \\
8 & MeSH descriptor: [Arthritis, Psoriatic] explode all trees & 199 \\
9 & psoriatic arthrit*ti,ab,kw (Word variations have been searched) & $55 \mid$ \\
10 & MeSH descriptor: [Medication Adherence] explode all trees & I,I4I \\
11 & MeSH descriptor: [Patient Compliance] explode all trees & 9,064 \\
12 & adher* near/5 (regimen* or medication* or treatment* or therap* or drug* or intervention* or biologic*):ti,ab,kw
\end{tabular}

13 ((complian* or complian* or complian*) near/5 (regimen* or medication* or treatment* or therap* or drug* or intervention* or biologic*)):ti,ab,kw (Word variations have been searched)

((persist*) near/5 (regimen* or medication* or treatment* or therap* or drug* or intervention* or biologic*)):ti,ab,kw

(Word variations have been searched) 
e-Pub search in PubMed, January 12, 2016

(((c((““Arthritis, Rheumatoid”[Mesh:noexp]) OR (“Rheumatoid Nodule”[Mesh:noexp]) OR (“Psoriasis"[Mesh:noexp]) OR ("Arthritis, Psoriatic"[Mesh:noexp]) OR (rheumatoid nodule*[tiab]) OR (arthritis rheumat*[tiab]) OR (RA[tiab]) OR (psoria*[tiab]) OR (psoriatic arthrit*[tiab]))))) AND ((()"Medication Adherence"[Mesh:noexp]) OR ("Patient Compliance"[Mesh:noexp]) OR ("Treatment Refusal"[Mesh:noexp]) OR ("Patient Dropouts"[Mesh:noexp]) OR (adher*[tiab]) OR (complian*[tiab]) OR (persist*[tiab]) OR (cooperat*[tiab]) OR (co-operat*[tiab]) OR (treatment refusal[tiab]) OR (patient dropout*[tiab]) OR (nonadher*[tiab]) OR (non-adher*[tiab]) OR (non-persist*[tiab]) OR (nonpersist*[tiab]) OR (noncomplian*[tiab]) OR (noncomplian*[tiab]) OR (uncooperat*[tiab]) OR (uncooperat*[tiab]) OR (patient perspective*[tiab]) OR (patient attitude*[tiab]) OR (patient experience*[tiab]) OR (continuation rate*[tiab]) OR (assessment adher*[tiab]) OR (assessment complian*[tiab]) OR (assessment persist*[tiab]) OR (assessment nonadher*[tiab]) OR (assessment nonadher*[tiab]) OR (assessment noncomplian*[tiab]) OR (assessment non-complian*[tiab]) OR (assessment nonpersist*[tiab]) OR (assessment non-persist*[tiab])))))) AND ((publisher[sb] NOT pubstatusnihms[All Fields] NOT pubstatuspmcsd[All Fields] NOT pmcbook[All Fields]) OR pubstatusaheadofprint[All Fields])

Search Result: 87

\section{Publish your work in this journal}

Patient Preference and Adherence is an international, peer-reviewed, open access journal that focuses on the growing importance of patient preference and adherence throughout the therapeutic continuum. Patient satisfaction, acceptability, quality of life, compliance, persistence and their role in developing new therapeutic modalities and compounds to optimize clinical outcomes for existing disease states are major areas of interest for the journal. This journal has been accepted for indexing on PubMed Central. The manuscript management system is completely online and includes a very quick and fair peer-review system, which is all easy to use. Visit http://www. dovepress.com/testimonials.php to read real quotes from published authors. 